\title{
HYPERANALYTIC PREDICATES
}

\author{
BY \\ YIANNIS N. MOSCHOVAKIS( $\left.{ }^{1}\right)$
}

Consider the two operations on number-theoretic predicates which allow us to (1) pass from a predicate $P\left(x_{1}, \ldots, x_{n}\right)$ to a predicate $Q\left(y_{1}, \ldots, y_{m}\right)$ recursive in $P\left(x_{1}, \ldots, x_{n}\right)$, and (2) pass from a predicate $P\left(y, x_{1}, \ldots, x_{n}\right)$ to the predicate (Ey) $P\left(y, x_{1}, \ldots, x_{n}\right)$. If we start with recursive predicates and apply these operations any finite number of times, we obtain the arithmetical hierarchy. If we now extend application of these operations into the transfinite (letting the predicates that we are defining determine, in any one of several equivalent ways, "how many times" these operations can be iterated) we obtain the hyperarithmetic hierarchy.

From this point of view the hyperarithmetic predicates are just those which can be defined "constructively" from recursive predicates, except for use of the existential number quantifier. This is made precise in Kleene's XLVIII of RFI $\left({ }^{2}\right)$, which asserts that a number-theoretic predicate is hyperarithmetic if and only if it is recursive in the type- 2 object ${ }^{2} \mathbf{E}$ that embodies number quantification,

$$
\begin{aligned}
{ }^{2} \mathbf{E}(\alpha) & =0 & & \text { if }(E t)[\alpha(t)=0], \\
& =1 & & \text { otherwise. }
\end{aligned}
$$

Kleene extends this approach in RFII to higher types, by calling a predicate $P\left(\alpha_{1}, \ldots, \alpha_{n}, x_{1}, \ldots, x_{m}\right)$ of number and function variables hyperanalytic, if it is recursive in the type-3 object ${ }^{3} \mathbf{E}$ which embodies function quantification,

$$
\begin{aligned}
{ }^{3} \mathbf{E}(\mathbf{F}) & =0 & & \text { if }(E \alpha)[\mathbf{F}(\alpha)=0], \\
& =1 & & \text { otherwise. }
\end{aligned}
$$

He then defines a hierarchy $H_{a}^{2}(\alpha)\left(a \in O^{2}\right)$ of hyperanalytic predicates, very similar to the hierarchy $H_{a}(x)(a \in O)$ of hyperarithmetic predicates, and asks if every hyperanalytic predicate is recursive in some $H_{a}^{2}(\alpha)$. Clarke studies this hierarchy in some detail in [1] and conjectures that it does not exhaust the hyperanalytic predicates.

In $\S 1$ we prove Clarke's conjecture. What seems to go wrong in the definition of $O^{2}$ and $H_{a}^{2}(\alpha)$, is that only countable ordinals (which are order-types of hyperanalytic well-orderings) are used, and in an effective manner. Since ${ }^{3} \mathbf{E}$ is powerful

Received by the editors February 11, 1966.

(1) During the preparation of this paper the author was partially supported by a grant from the National Science Foundation.

$\left({ }^{2}\right)$ References to the bibliography are by number, except for Kleene's [3], [6] and [7] which we call IM, RFI, and RFII respectively. 
enough to allow us to refer to the totality of countable ordinals, we are able to diagonalize through $O^{2}$ and define a hyperanalytic predicate, recursive in no $H_{a}^{2}(\alpha)$.

In this paper we define and study a hierarchy which exhausts the class of hyperanalytic predicates. Our definition is a generalized induction (like those of $O$ in [4] and $\{z\}(c) \simeq w$ in RFI) whose classical interpretation as a transfinite induction employs uncountable ordinals. (Since there are only countably many hyperanalytic predicates, our definition yields a hierarchy indexed by countable ordinals, in fact, exactly those which are order-types of hyperanalytic well-orderings; but this is "after the fact," and is just noneffective enough to prohibit diagonalization.)

A number-theoretic predicate is hyperarithmetic if and only if both it and its negation are $\Pi_{1}^{1}$, i.e., expressible in the form $(\alpha) A\left(\alpha, x_{1}, \ldots, x_{n}\right)$, with $A$ arithmetical. The correct analog of $\Pi_{1}^{1}$ in type 3 seems to be recursively enumerable in ${ }^{3} \mathbf{E}$, i.e., expressible in the form " $\mathrm{f}\left(\alpha_{1}, \ldots, \alpha_{n}, x_{1}, \ldots, x_{m}\right)$ is defined," with $\mathrm{f}$ partial recursive in ${ }^{3} \mathbf{E}$. In $\$ \$ 4,5$ we prove some elementary properties of predicates r.e. in ${ }^{3} \mathbf{E}$, including the analog of the statement above, several "choice axioms" and the fact that this class of predicates is closed under number quantification. These results are inspired by Gandy's [2], where similar theorems are used to study the theory of hyperarithmetic predicates relativized to an arbitrary type- 2 object.

In $\S 6$ we give a slight improvement of Kleene's representation theorems for the predicate $\{z\}(c) \simeq w$ (for type-3), and we prove that the class of predicates r.e. in ${ }^{3} \mathbf{E}$ is not closed under existential function quantification. This leads us in $\$ 7$ to a study of a hierarchy that extends the hyperanalytic predicates and is similar in many respects to the hierarchy of $\Delta_{2}^{1}$ predicates.

We use the notation and terminology of Kleene's RFI and RFII, with very few exceptions. However the only results about recursive functionals that are essential are those in $\S \S 1-4$ of RFI. We quote here a version of Kleene's XXII in RFI, which we shall find very useful.

KleEne's Substitution Lemma. There are primitive recursive functions $\gamma_{1}(z, w)$ and $\gamma_{2}(z, w)$ such that for a list of variables $\mathfrak{c}$ of types $\leqq 3$ :

(1) $\left\{\gamma_{1}(e, a)\right\}(\mathfrak{c})=\{e\}(\mathfrak{c}, \lambda t\{a\}(\mathfrak{c}, t))$, when $\lambda t\{a\}(\mathfrak{c}, t)$ is completely defined and $\{e\}(\mathfrak{c}, \lambda t\{a\}(\mathfrak{c}, t))$ is defined.

(2) $\left\{\gamma_{2}(e, a)\right\}(\mathfrak{c})=\{e\}(\mathfrak{c}, \lambda \alpha\{a\}(\mathfrak{c}, \alpha))$, when $\lambda \alpha\{a\}(\mathfrak{c}, \alpha)$ is completely defined and $\{e\}(c, \lambda \alpha\{a\}(c, \alpha))$ is defined.

We thank the referee for discovering a host of minor errors (the remaining host is our own responsibility) as well as a more serious error in the proof of Theorem 6.

This paper was presented to the Association of Symbolic Logic at the April 4, 1966 meeting in New York. The abstract from that talk (to appear in the Journal of Symbolic Logic) gives a concise summary of the most interesting results in the paper and can serve as a more technical introduction than our preceding remarks.

1. A hyperanalytic function not recursive in any $H_{a}^{2}(\alpha)$. The set $O^{2}$ and the predicates $H_{a}^{2}(\alpha)\left(a \in O^{2}\right)$ are defined in $\S 11$ of RFII. 
LEMMA 1 (IMPLICIT IN RFII). There is a primitive recursive function $\mathrm{g}(a)$, such that if $a \in O^{2}$, then $\lambda \alpha\{\mathrm{g}(a)\}\left({ }^{3} \mathbf{E}, \alpha\right)$ is completely defined and

$$
H_{a}^{2}(\alpha) \equiv\{\mathbf{g}(a)\}\left({ }^{3} \mathbf{E}, \alpha\right)=0 .
$$

For an arbitrary function $\xi$, set

$$
\begin{gathered}
D_{\xi}=\{x: \xi(\langle 1, x\rangle)=0\}, \\
x \leqq_{\xi} y \equiv \xi(\langle x, y\rangle)=0\left(^{3}\right) .
\end{gathered}
$$

We say that $\xi$ is a well-ordering, $\xi \in \mathrm{WO}$, if the following three conditions are satisfied:

(1) $\leqq_{\xi}$ is a well-ordering on $D_{\xi}$ with minimum 1 .

(2) For each $x \in D_{\xi}, 2^{x}$ is the successor of $x$ in $D_{\xi}$.

(3) For each $x \in D_{\xi}, x$ is a limit point of $\leqq_{\xi}$ if and only if $x=3^{(x)}{ }_{1},(x)_{1} \neq 0$.

It is clear that the predicate $\xi \in \mathrm{WO}$ is analytic, hence recursive in ${ }^{3} \mathbf{E}$.

LEMMA 2. Let

$$
\begin{aligned}
P(\xi, \alpha, x) \equiv & \xi \in \text { WO \& } x \in D_{\xi} \&[\alpha \text { is a similarity mapping from the } \\
& \text { initial segment of } \leqq_{\xi} \text { up to and including } x \text { onto some initial } \\
& \text { segment of the partial ordering } \left.<_{0}^{2}\right] .
\end{aligned}
$$

Then $P(\xi, \alpha, x)$ is hyperanalytic, i.e. recursive in ${ }^{3} \mathbf{E}$.

Proof. We shall define the characteristic function $\mathrm{f}(\xi, \alpha, x)$ of $P(\xi, \alpha, x)$ using the Recursion Theorem XIV of RFI. Thus, we shall define a partial recursive function $\mathrm{h}\left(f,{ }^{3} \mathbf{E}, \xi, \alpha, x\right)$, then seek a number $f$ such that

$$
\mathrm{h}\left(f,{ }^{3} \mathbf{E}, \xi, \alpha, x\right) \simeq\{f\}\left({ }^{3} \mathbf{E}, \xi, \alpha, x\right)
$$

and set

$$
\mathrm{f}(\xi, \alpha, x) \simeq\{f\}\left({ }^{3} \mathbf{E}, \xi, \alpha, x\right) .
$$

To simplify notation, here and in similar cases in the future, we avoid the symbols " $\mathrm{h}\left(f,{ }^{3} \mathbf{E}, \xi, \alpha, x\right)$ " and " $\{f\}\left({ }^{3} \mathbf{E}, \xi, \alpha, x\right)$ " and simply use " $\mathrm{f}(\xi, \alpha, x)$ " as synonymous with these, even before we complete the definition of $\mathrm{h}\left(f,{ }^{3} \mathbf{E}, \xi, \alpha, x\right)$ and the application of the recursion theorem.

$\left({ }^{3}\right)$ We follow the notation of IM, RFI and RFII, with only one addition: for a partial function $\mathbf{f}(\mathrm{c}), \mathbf{f}(\mathrm{c}) \downarrow \equiv \mathbf{f}(\mathrm{c})$ is defined.

The primitive recursive functions $(a)_{i}, 1 \mathrm{~h}(a)$ and $a^{*} b$ are defined in $\$ 45$ of IM. Other notations that we often use are:

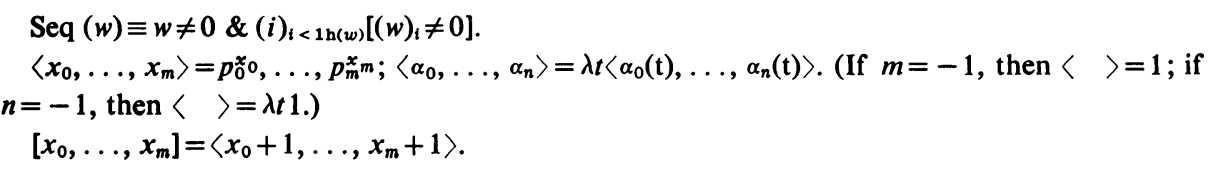


After $\mathrm{f}(\xi, \alpha, x)$ has been defined as a partial function, we shall prove that it is completely defined and that it is the characteristic function of $P(\xi, \alpha, x)$.

The definition of $\mathrm{f}(\xi, \alpha, x)$ from its index $f$ is by cases, using XVIII of RFI. As in several later constructions, the partial recursive predicates which determine the cases are quite complicated; we believe that producing explicit formulas for them would only conceal our motivation. Instead, we give intuitive instructions for the computations to be performed, from which the explicit formulas can be derived by routine applications of the results in $\$ \S 1-4$ of RFI.

Since the cases split into subcases and these further into sub-subcases etc., we use a numbering which follows this "tree structure." Thus, if there are three subcases of case 121 we call them 1211, 1212 and 1213. After the number of a case we identify in brackets the case hypothesis.

In some instances we ask that the value of a certain partial predicate or function be computed, and then the main computation splits into subcases according to the value obtained (if any). We label these branching points in the computation "questions" and prefix their numbers with a "?," e.g. ?122 below, to be read "question 122." Sometimes, when this reflects our motivation, we phrase the instructions at these branching points as questions to the oracle ${ }^{3} \mathbf{E}$. Thus ?1 below "ask ${ }^{3} \mathbf{E}$ if $\xi \in \mathrm{WO} \& x \in D_{\xi}$ " means "go to 12 if $\xi \in \mathrm{WO} \& x \in D_{\xi}$, go to 11 if not $\left(\xi \in \mathrm{WO} \& x \in D_{\xi}\right)$," where the predicate $\xi \in \mathrm{WO} \& x \in D_{\xi}$ is recursive in ${ }^{3} \mathbf{E}$.

Instructions for the computation of $\mathrm{f}(\xi, \alpha, x)$ :

?1. Ask ${ }^{3} \mathrm{E}$ if $\xi \in \mathrm{WO} \& x \in D_{\xi}$.

11 [No to ?1]. Give output 1.

12 [Yes to ?1]. Go to 121,122 or 123 according as $\left.x=1,\left[x=2^{(x)}\right)_{0} \&(x)_{0} \neq 0\right]$ or $x=3^{(x)}{ }_{1}\left((x)_{1} \neq 0\right)$. If none of these cases applies, give output 1 .

$121[x=1]$. If $\alpha(1)=1$, give output 0 . If $\alpha(1) \neq 1$, give output 1 .

?122 $\left[x=2^{y}\right.$, where $\left.y=(x)_{0} \neq 0\right]$. Compute $\mathrm{f}(\xi, \alpha, y)$.

$1221\left[\mathrm{f}(\xi, \alpha, y) \simeq 0\right.$ in ?122]. If $\alpha(x)=2^{\alpha(y)}$, give output 0 , otherwise give output 1 .

$1222[\mathrm{f}(\xi, \alpha, y) \simeq 1$ in ?122]. Give output 1.

?123 $\left[x=3^{w}\right.$, where $\left.w=(x)_{1} \neq 0\right]$. Ask ${ }^{3} \mathbf{E}$ if $\alpha(x)=3.5^{(\alpha(x))_{2}} 2.7^{(\alpha(x))_{3}}$

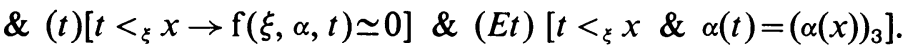

1231 [No to ?123]. Give output 1.

?1232 [Yes to ?123; let $y=(\alpha(x))_{2}, u=(\alpha(x))_{3}$ ]. Ask ${ }^{3} \mathbf{E}$ if

[ $y$ defines $y_{n}$ general recursively from $\lambda \alpha\{g(u)\}\left({ }^{3} \mathbf{E}, \alpha\right)$ as a function of $n_{O}$ ] \& $y_{0}=u$ $\&(n)(E s)(E t)\left[s<_{\xi} t \quad \& \quad t<_{\xi} x \quad \& \quad \alpha(s)=y_{n} \quad \& \quad \alpha(t)=y_{n+1}\right]$.

12321 [No to ?1232]. Give output 1.

12322 [Yes to ?1232]. Give output 0.

We must now verify that each of the questions above is legitimate, i.e., that we are indeed asking for the computation of a predicate or function which is partial recursive in ${ }^{3} \mathbf{E}$.

In $? 1$ this is obvious, and in ?122 we ask for a computation which can be performed from the index $f$ of $\mathrm{f}(\xi, \alpha, x)$. 
In ?123 we have a tacit application of the Subsitution Lemma. The predicate

$$
\begin{aligned}
Q(\xi, \alpha, x, \beta) \equiv \alpha(x) & =3 \cdot 5^{(\alpha(x))_{2}} \cdot 7^{(\alpha(x))_{3}} \\
& \&(t)[\beta(t)=0] \\
& \&(E t)\left[t<_{\xi} x \& \alpha(t)=(\alpha(x))_{3}\right]
\end{aligned}
$$

is clearly recursive in ${ }^{3} \mathbf{E}$. We wish to evaluate $Q(\xi, \alpha, x, \beta)$ for

$$
\begin{aligned}
\beta(t) & \simeq 0 \text { if } \overline{t<_{\xi} x} \\
& \simeq 0 \text { if } t<_{\xi} x \& \mathrm{f}(\xi, \alpha, x) \simeq 0, \\
& \simeq 1 \text { if } t<_{\xi} x \& \mathrm{f}(\xi, \alpha, x) \simeq 1 .
\end{aligned}
$$

By the Substitution Lemma, there is a partial recursive $Q^{\prime}\left({ }^{3} \mathbf{E}, \xi \alpha, x\right)$ which agrees with $Q(\xi, \alpha, x, \beta)$ for this $\beta$, if $\beta$ is completely defined. It is this $Q^{\prime}\left({ }^{3} \mathbf{E}, \xi, \alpha, x\right)$ that we are instructed to evaluate in ?123. (Notice that as before we are suppressing the dependence of $Q^{\prime}\left({ }^{3} \mathbf{E}, \xi, \alpha, x\right)$ on $f$.)

A similar tacit application of the Substitution Lemma occurs in ?1232, where the substitution is for a type- 2 object. The clause [ $y$ defines $y_{n}$ general recursively from $F$ as a function of $n_{O}$ ] is recursive in ${ }^{3} \mathbf{E}$, as a predicate of $y$ and a type-2 object F. Since the other clauses are clearly recursive in ${ }^{3} \mathbf{E}$, we interpret 21232 as instructions to evaluate a certain recursive $R\left({ }^{3} \mathbf{E}, \xi, \alpha, x, \mathbf{F}\right)$, for $\mathbf{F}=\lambda \alpha\{g(u)\}\left({ }^{3} \mathbf{E}, \alpha\right)$. The Substitution Lemma gives us a partial recursive $R^{\prime}\left({ }^{3} \mathbf{E}, \xi, \alpha, x\right)$ which agrees with $R\left({ }^{3} \mathbf{E}, \xi, \alpha, x, \mathbf{F}\right)$ for $\mathbf{F}=\lambda \alpha\{g(u)\}\left({ }^{3} \mathbf{E}, \alpha\right)$, if $\lambda \alpha\{g(u)\}\left({ }^{3} \mathbf{E}, \alpha\right)$ is completely defined, and it is this $R^{\prime}\left({ }^{3} E, \xi, \alpha, x\right)$ that we evaluate.

In the more complicated computation instructions of $\$ 4$ we shall let the reader supply these tacit references to the Substitution Lemma. We hope that the detailed analyses of these two cases above are sufficiently clear to make the process routine.

If $\xi \in$ WO \& $x \in D_{\xi}$ is false, then $\mathrm{f}(\xi, \alpha, x) \simeq 1$ by case 11 .

If $\xi \in$ WO \& $x \in D_{\xi}$ let $|x|_{\xi}$ be the order type of the initial segment $[1, x]_{\xi}$ of $\leqq_{\xi}$ up to and including $x$. We prove that $\mathrm{f}(\xi, \alpha, x)$ is defined and agrees with the characteristic function of $P(\xi, \alpha, x)$ by transfinite induction on $|x|_{\xi}$.

If $x=1, \mathrm{f}(\xi, \alpha, x)$ is defined and has the correct value by case 121 .

If $x=2^{y} \quad\left(y=(x)_{0}\right)$, we may assume by the induction hypothesis that for each $t \leqq_{\xi} y, \mathrm{f}(\xi, \alpha, t)$ is defined and has the correct value. In particular ?122 is answered and the computation shifts to either 1221 or 1222 , whence the correct value is obtained.

If $x=3^{w}\left(w=(x)_{1}\right)$, we may assume the induction hypothesis for each $t<_{\xi} x$. Thus ?123 with the interpretation given it above is answered, since $\beta$ is completely defined. If $\alpha(x)$ is not of the proper form, or if $\alpha$ does not map each $[1, t]_{\xi}(t<x)$ onto some initial segment of $<_{0}^{2}$, we give the correct output 1 by 1231 . If these conditions are satisfied, we further ask ?1232. Since by induction hypothesis $u=\alpha(t) \in O^{2}, \lambda \alpha\{\mathrm{g}(u)\}\left({ }^{3} \mathbf{E}, \alpha\right)$ is completely defined and hence ?1232 is answered correctly in the way it was interpreted, whence we are led to either 12321 or 12322 and the correct output. 
THEOREM 1. There exists a hyperanalytic function $\mathrm{f}(x)$ which is not recursive in any $H_{a}^{2}(\alpha)\left(a \in O^{2}\right)$.

Proof. Since

$$
a \in O^{2} \equiv(E \xi)(E \alpha)(E x)[P(\xi, \alpha, x) \& \alpha(x)=a],
$$

the set $O^{2}$ is hyperanalytic. Then the predicate

$$
\begin{aligned}
Q(\alpha) & \equiv \alpha(0) \in O^{2} \& H_{\alpha(0)}^{2}(\lambda t \alpha(t+1)) \\
& \equiv \alpha(0) \in O^{2} \&\{g(\alpha(0))\}\left({ }^{3} \mathbf{E}, \lambda t \alpha(t+1)\right)=0
\end{aligned}
$$

is hyperanalytic. Now each $H_{a}^{2}(\alpha)$ is recursive in $Q(\alpha)$, hence $Q(\alpha)$ cannot be recursive in any $H_{b}^{2}(\alpha)$; because then $H_{2}^{2} b(\alpha)$ would be recursive in $H_{b}^{2}(\alpha)$, which is impossible.

Put

$$
\begin{aligned}
f(e, \mathbf{F}, t) & \simeq 0 & & \text { if }\{e\}(\mathbf{F}, t) \text { is not defined } \\
& \simeq\{e\}(\mathbf{F}, t)+1 & & \text { if }\{e\}(\mathbf{F}, t) \text { is defined }
\end{aligned}
$$

and let

$$
\mathrm{f}(t)=\mathrm{f}(t, \lambda \alpha \mathrm{q}(\alpha), t),
$$

where $\mathrm{q}(\alpha)$ is the characteristic function of $Q(\alpha)$. It is easy to verify that $\mathrm{f}(t)$ is hyperanalytic but not recursive in $Q(\alpha)$, hence not recursive in any $H_{a}^{2}(\alpha)$.

2. Functions recursive in a type-3 object. Let $\tau^{2}$ and $\tau^{3}$ be given objects of types 2 and 3 respectively. For each number-theoretic function $\tau$ we shall define a set of numbers $N\left(\tau^{3}, \tau^{2}, \tau\right)=N(\tau)$, and for each $z \in N(\tau)$ a number-theoretic function $\mathrm{f}_{z}^{\tau^{3}, \tau^{2}, \tau}(t)=\mathrm{f}_{z}^{\tau}(t)$.

The definition will be by induction and will be given simultaneously for all $\tau$. Thus it is most properly viewed as an inductive definition of a predicate $\lambda z \tau z \in N(\tau)$ and a partial functional $\lambda z \tau t \mathrm{f}_{z}^{\tau}(t)$, defined when $z \in N(\tau)$.

For typographical reasons we often write $\mathrm{f}(\tau, z ; t)$ or $\mathrm{f}^{\tau}(z ; t)$ for $\mathrm{f}_{z}^{\tau}(t)$.

Definition 1. (1) For each $q,\langle 1, q\rangle \in N(\tau)$ and $\mathrm{f}_{\langle 1, q\rangle}^{\tau}(t)=q$. (Introduction of constants.)

(2) $\langle 2,0\rangle \in N(\tau)$ and $\mathrm{f}_{\langle 2,0\rangle}^{\tau}(t)=\tau(t)$. (Introduction of $\tau$.)

(3) If $w \in N(\tau)$, then $\langle 3, w\rangle \in N(\tau)$ and $\mathrm{f}_{\langle 3, w\rangle}^{\tau}(t)=\tau^{2}\left(\lambda u \mathrm{f}_{w}^{\tau}(u)\right)$. (Introduction of $\tau^{2}$.)

(4) For $w \in N(\tau)$ and $e$ any number, put $e(\tau, w, t) \simeq\{e\}^{\alpha}(t)$, where $\alpha=\lambda u f_{w}^{\tau}(u)$.

If $w \in N(\tau)$ and if for each $t, e(\tau, w, t) \downarrow$ and $e(\tau, w, t) \in N(\tau)\left({ }^{3}\right)$, then $\langle 4, w, e\rangle \in$ $N(t)$ and $\mathrm{f}_{\langle 4, w, e\rangle}^{\tau}(t)=\mathrm{f}_{e(\tau, w, t)}^{\tau}(\mathrm{t})$. (Diagonalization.)

(5) Suppose that for each $\alpha$ and each $t,\{e\}^{\alpha, \tau}(t) \downarrow$; we say that $e$ defines a $\tau$ recursive functional $\{e\}(\alpha, \tau)=\lambda t\{e\}^{\alpha, \tau}(t)$.

If $e$ defines a $\tau$-recursive functional $\{e\}(\alpha, \tau)$ and if for each $\alpha, w \in N(\{e\}(\alpha, \tau))$, then $\langle 5, w, e\rangle \in N(\tau)$ and $\mathrm{f}_{\langle 5, w, e\rangle}^{\tau}(t)=\tau^{3}(\lambda \alpha \mathrm{f}(\{e\}(\alpha, \tau), w ; t))$. (Introduction of $\tau^{3}$.) 
(6) Suppose that for each $t,\{e\}^{\tau}(t) \downarrow$, put $\alpha=\lambda t\{e\}^{\tau}(t)$. If $w \in N(\alpha)$, then $\langle 6, w, e\rangle \in$ $N(\tau)$ and $\mathrm{f}_{\langle 6, w, e\rangle}^{\tau}(t)=\mathrm{f}_{w}^{\alpha}(t)$. (Transfer.)

(7) Recursion clause: $z \in N(\tau)$ and $\mathrm{f}_{z}^{\tau}(t)=u$ only by (1)-(6).

The main result of this section is that the class of functions $\lambda t f_{z}^{\tau}(t)(z \in N(\tau))$ coincides with the class of functions recursive in $\tau^{3}, \tau^{2}, \tau$.

Classically one interprets inductions of this type as definitions by transfinite induction over an initial segment of the ordinals. Clause (5) raises the possibility that in this case the induction extends into the third number class; in any case, we only use ordinals which are limits of fundamental sequences of cardinality not exceeding that of the continuum. We assign to each $z, \tau$ such that $z \in N(\tau)$ the ordinal $|z|^{\tau^{3}, \tau^{2}, \tau}=|z|^{\tau}$ at which we verify that $z \in N(\tau)$. The definition is by induction following the inductive definition of $z \in N(\tau)$.

DEFINITION 2. (1) $|\langle 1, q\rangle|^{\imath}=0$.

(2) $|\langle 2,0\rangle|^{\mathfrak{r}}=0$.

(3) $|\langle 3, w\rangle|^{\imath}=|w|^{\tau}+1$.

(4) $|\langle 4, w, e\rangle|^{\tau}=$ supremum $_{t}\left\{|w|^{\tau}+1,|e(\tau, w, t)|^{\tau}+1\right\}$.

(5) $|\langle 5, w, e\rangle|^{\tau}=$ supremum $_{\alpha}\left\{|w|^{(e\}(\alpha, \tau)}+1\right\}$.

(6) $|\langle 6, w, e\rangle|^{\tau}=|w|^{\alpha}+1$, where $\alpha=\lambda t\{e\}^{\tau}(t)$.

Whenever possible we prefer to give proofs or definitions by induction on $z \in N(\tau)$ rather than by transfinite induction on $|z|^{\tau}$. One can, of course, interpret all such arguments as classical transfinite inductions.

THEOREM 2. There is a primitive recursive function $\mathrm{p}(z)$, such that if $z \in N(\tau)$, then

$$
\mathrm{f}_{z}^{\tau}(t) \simeq\{\mathrm{p}(z)\}\left(\tau^{3}, \tau^{2}, \tau, t\right)
$$

Thus each $\mathrm{f}_{z}^{\tau}(t)$ is recursive in $\tau^{3}, \tau^{2}, \tau$.

Proof. We first define $\mathrm{p}(z)$ as a partial recursive function from a Gödel number $p$ using the recursion theorem, and then observe that it is totally defined and indeed primitive recursive. The proof that $\mathrm{p}(z)$ has the desired property will be by induction on $z \in N(\tau)$. We treat the most interesting cases right in the definition.

Case (1). $z=\langle 1, q\rangle$ for some $q$. Put $\mathrm{p}(z)=\langle 2,\langle 1,1,1,1\rangle, q\rangle$.

Case (2). $z=\langle 2,0\rangle$. Put $\mathrm{p}(z)=\langle 7,\langle 1,1,1,1\rangle\rangle$.

Case (3). $z=\langle 3, w\rangle$ for some $w$. Choose a primitive recursive $\mathrm{g}_{1}(e)$ such that for each $\tau, u, t,\left\{g_{1}(e)\right\}\left(\tau^{3}, \tau^{2}, \tau, u, t\right) \simeq\{e\}\left(\tau^{3}, \tau^{2}, \tau, u\right)$ and put

$$
\mathrm{p}(z)=\left\langle 8,\langle 1,1,1,1\rangle, 2, \mathrm{~g}_{1}(\mathrm{p}(w))\right\rangle .
$$

If the induction hypothesis is satisfied, we know that for each $u$,

Thus

$$
\{\mathrm{p}(w)\}\left(\tau^{3}, \tau^{2}, \tau, u\right) \simeq \mathrm{f}_{w}^{\tau}(u) .
$$

$$
\begin{aligned}
\{\mathrm{p}(z)\}\left(\tau^{3}, \tau^{2}, \tau, t\right) & \simeq \tau^{2}\left(\lambda u\left\{\mathrm{~g}_{1}(\mathrm{p}(w))\right\}\left(\tau^{3}, \tau^{2}, \tau, u, t\right)\right) \\
& \simeq \tau^{2}\left(\lambda u\{\mathrm{p}(w)\}\left(\tau^{3}, \tau^{2}, \tau, u\right)\right) \simeq \tau^{2}\left(\lambda u \mathrm{f}_{w}^{\tau}(u)\right) \simeq \mathrm{f}_{z}^{\tau}(t)
\end{aligned}
$$


Case (4). $z=\langle 4, w, e\rangle$ for some $w, e$. The functional

$$
\phi\left(\tau^{3}, \tau^{2}, \tau, \alpha, p, e, t\right) \simeq\left\{\mathrm{p}\left(\{e\}^{\alpha}(\mathrm{t})\right)\right\}\left(\tau^{3}, \tau^{2}, \tau, t\right)
$$

is evidently partial recursive. Using the Substitution Lemma, choose a primitive recursive $\mathrm{g}_{2}(p, w, e)$ such that

$$
\left\{\mathrm{g}_{2}(p, w, e)\right\}\left(\tau^{3}, \tau^{2}, \tau, t\right)=\phi\left(\tau^{3}, \tau^{2}, \tau, \alpha, p, e, t\right),
$$

when $\alpha=\lambda u\{\mathrm{p}(w)\}\left(\tau^{3}, \tau^{2}, \tau, u\right)$ is completely defined and $\phi\left(\tau^{3}, \tau^{2}, \tau, \alpha, p, e, t\right)$ is defined. Put $\mathrm{p}(z)=\mathrm{g}_{2}(p, w, e)$.

If the induction hypothesis is satisfied, then $\alpha$ is completely defined, and for each $t,\{e\}^{\alpha}(t) \downarrow \&\left\{\mathrm{p}\left(\{e\}^{\alpha}(t)\right)\right\}\left(\tau^{3}, \tau^{2}, \tau, t\right) \simeq \mathrm{f}_{z}^{\tau}(t)$, so that $\mathrm{p}(z)$ has the required property.

Case (5). $z=\langle 5, w, e\rangle$ for some $w, e$. Using the Substitution Lemma, choose a primitive recursive $\mathrm{g}_{3}(p, w, e)$ such that

$$
\left\{\mathrm{g}_{3}(p, w, e)\right\}\left(\tau^{3}, \tau^{2}, \alpha, \tau, t\right)=\{\mathrm{p}(w)\}\left(\tau^{3}, \tau^{2},\{e\}(\alpha, \tau), t\right),
$$

when $\{e\}(\alpha, \tau)=\lambda u\{e\}^{\alpha, \tau}(u)$ is completely defined and the right-hand side of the equation is defined. Put $\mathrm{p}(z)=\left\langle 8,\langle 1,1,1,1\rangle, 3, \mathrm{~g}_{3}(p, w, e)\right\rangle$.

If the induction hypothesis is satisfied, then for each $\alpha,\{e\}(\alpha, \tau)$ is completely defined and $\{\mathrm{p}(w)\}\left(\tau^{3}, \tau^{2},\{e\}(\alpha, \tau), t\right)=\mathrm{f}(\{e\}(\alpha, \tau), w ; t)$, so that

$$
\{\mathrm{p}(z)\}\left(\tau^{3}, \tau^{2}, \tau, t\right) \simeq \tau^{3}(\lambda \alpha \mathrm{f}(\{e\}(\alpha, \tau), w ; t)) .
$$

Case (6). $z=\langle 6, w, e\rangle$ for some $w, e$. Using the Substitution Lemma, choose a primitive recursive $\mathrm{g}_{4}(p, w, e)$ such that

$$
\left\{\mathrm{g}_{4}(p, w, e)\right\}\left(\tau^{3}, \tau^{2}, \tau, t\right)=\{\mathrm{p}(w)\}\left\{\tau^{3}, \tau^{2}, \lambda u\{e\}^{\tau}(u), t\right),
$$

when $\lambda u\{e\}^{\tau}(u)$ is completely defined and the right-hand side is defined. Put $\mathrm{p}(z)=\mathrm{g}_{4}(p, w, e)$.

Case (7). Otherwise. Put $\mathrm{p}(z)=0$.

To simplify notation for the next theorem we introduce the conventions

$$
\boldsymbol{x}=x_{1}, \ldots, x_{m} ; \quad \tau=\tau_{1}, \ldots, \tau_{n} .
$$

(Now $[\boldsymbol{x}]=\left[x_{1}, \ldots, x_{m}\right]$ and $\langle\tau\rangle=\left\langle\tau_{1}, \ldots, \tau_{n}\right\rangle$ are defined in $\left({ }^{3}\right)$, including the case for empty $x$ or $\tau$.)

THEOREM 3. There is a primitive recursive function $\mathrm{q}(e, x)$ such that:

(1) $\{e\}\left(\tau^{3}, \tau^{2}, \tau, x\right) \downarrow \equiv \mathrm{q}(e,[x]) \in N(\langle\tau\rangle)$.

(2) $\{e\}\left(\tau^{3}, \tau^{2}, \tau, x\right) \simeq w \rightarrow(t)[\mathrm{f}(\langle\tau\rangle, \mathrm{q}(e,[x]) ; t)=w]$.

Proof. For this proof only, let us introduce the additional notation conventions:

$$
\begin{gathered}
\{e\}(\tau, \boldsymbol{x}) \simeq\{e\}\left(\tau^{3}, \tau^{2}, \boldsymbol{\tau}, \boldsymbol{x}\right) . \\
x=[\boldsymbol{x}]=\left[x_{1}, \ldots, x_{m}\right] ; \quad \tau=\langle\tau\rangle=\left\langle\tau_{1}, \ldots, \tau_{n}\right\rangle .
\end{gathered}
$$

We shall define $\mathrm{q}(e, x)$ from its Gödel number $q$ using the recursion theorem in the usual fashion. The definition will be by cases on $e$ being an index of the proper 
kind for $x$. We number the cases S1, S2, etc. and give as case hypothesis the definition of $\{e\}(\tau, x)$ rather than the number-theoretic condition that $e$ and $x$ must satisfy. For example, Case $\mathrm{S} 1$ below, $\{e\}(\tau, x)=x_{1}^{\prime}$, should be described by

$$
\text { Seq }(x) \&(E n)[e=\langle 1,\langle 1 \mathrm{~h}(x), n, 1,1\rangle\rangle] \text {. }
$$

The implication from left to right in (1) together with (2) of the theorem are proved by induction on $\{e\}(\tau, x) \simeq w$. We treat the most interesting cases right in the definition. Proof of the implication from right to left in (1) is by induction on $\mathrm{q}(e, x) \in N(\tau)$ and will be outlined after the definition is completed.

Case S1. $\{e\}(\tau, x)=x_{1}^{\prime}$. Choose a primitive recursive $\mathrm{r}_{1}(x)$ such that for each $\alpha, x, t,\left\{\mathrm{r}_{1}(x)\right\}^{\alpha}(t)=\left\langle 1,(x)_{0}\right\rangle$, and put $\mathrm{q}(e, x)=\left\langle 4,\langle 2,0\rangle, \mathrm{r}_{1}(x)\right\rangle$. Clearly $\mathrm{q}(e, x) \in N(\tau)$ and $\mathrm{f}(\tau, \mathrm{q}(e, x) ; t)=\mathrm{f}\left(\tau,\left\langle 1,(x)_{0}\right\rangle ; t\right)=x_{1}^{\prime}$.

Case S2. $\{e\}(\tau, \boldsymbol{x})=k$. Put $\mathrm{q}(e, x)=\langle 1, k\rangle$.

Case S3. $\{e\}(\tau, x)=x_{1}$. Treat similarly to Case S1.

Case S4. $\{e\}(\tau, x) \simeq\{g\}(\{h\}(\tau, x), \tau, x)$. Choose a primitive recursive $\mathrm{r}_{2}(q, g, x)$ such that for each $\alpha, t,\left\{\mathrm{r}_{2}(q, g, x)\right\}^{\alpha}(t) \simeq\{q\}(g,[\alpha(0)] * x)$. Put

$$
\mathrm{q}(e, x)=\left\langle 4, \mathrm{q}(h, x), \mathrm{r}_{2}(q, g, x)\right\rangle .
$$

Assume that $\{h\}(\tau, x) \simeq u \&\{g\}(u, \tau, x) \simeq w$. By induction hypothesis, $\mathrm{q}(h, x) \in$ $N(\tau), \mathrm{f}^{\tau}(\mathrm{q}(h, x) ; t)=u$ (all $\left.t\right), \mathrm{q}(g,[u] * x) \in N(\tau)$ and $\mathrm{f}^{\tau}(\mathrm{q}(g,[u] * x) ; t)=w$ (all $\left.t\right)$. Thus $\mathrm{q}(e, x) \in N(\tau)$, since $\mathrm{q}(h, x) \in N(\tau)$ and for $\alpha=\lambda u \mathrm{f}^{\tau}(\mathrm{q}(h, x) ; u)$ and each $t$,

$$
\left\{\mathrm{r}_{2}(q, g, x)\right\}^{\alpha}(t) \simeq\{q\}(g,[\alpha(0)] * x) \simeq \mathrm{q}(g,[u] * x) \in N(\tau) .
$$

Moreover, $\mathrm{f}^{\mathrm{t}}(\mathrm{q}(e, x) ; t)=\mathrm{f}^{\mathrm{\tau}}(\mathrm{q}(g,[u] * x) ; t)=w$.

Case S5. Subcase (a). $\{\mathrm{e}\}(\tau, 0, x) \simeq\{g\}(\tau, x)$. Choose a primitive recursive $\mathrm{r}_{3}(q, g, x)$ such that for each $\alpha, t,\left\{\mathrm{r}_{3}(q, g, x)\right\}^{\alpha}(t) \simeq\{q\}(g, x)$ and put $\mathrm{q}(e,[0] * x)=$ $\left\langle 4,\langle 2,0\rangle, \mathrm{r}_{3}(q, g, x)\right\rangle$.

Assume that $\{g\}(\tau, x) \simeq w$. By induction hypothesis, $\mathrm{q}(g, x) \in N(\tau)$ and for each $t$, $\mathrm{f}^{t}(\mathrm{q}(g, x) ; t)=w$. Since $\langle 2,0\rangle \in N(\tau)$ and since for each $\alpha$,

$$
\left\{\mathrm{r}_{3}(q, g, x)\right\}^{\alpha}(t) \simeq \mathrm{q}(g, x) \in N(\tau),
$$

we have $\mathrm{q}(e,[0] * x) \in N(\tau)$ and $\mathrm{f}^{\tau}(\mathrm{q}(e,[0] * x) ; t)=\mathrm{f}^{\tau}(\mathrm{q}(g, x) ; t)=w$.

REMARK. The simpler definition $\mathrm{q}(e,[0] * x)=\mathrm{q}(g, x)$ would serve equally well for this part of the theorem. In proving the implication from right to left in (1) however, this definition would not immediately give us a usable induction hypothesis. With the definition we chose, if $\mathrm{q}(e,[0] * x) \in N(\tau)$, then $\mathrm{q}(g, x) \in N(\tau)$ and $\mathrm{q}(g, x)$ is a predecessor of $\mathrm{q}(e,[0] * x)$ in the definition of $N(\tau)$ (in particular $|\mathrm{q}(g, x)|^{\tau}$ $\left.<|q(e,[0] * x)|^{\imath}\right)$. Thus in treating this case of the inductive proof of the right-to-left implication in (1), the induction hypothesis assures us that $\{g\}(\tau, x) \downarrow$, which implies immediately that $\{e\}(\tau, 0, x) \downarrow$.

Case S5. Subcase (b). $\{e\}(\tau, y+1, x) \simeq\{h\}(y,\{e\}(\tau, y, x), \tau, x)$. Choose a primitive recursive $\mathrm{r}_{4}(q, h, y, x)$ such that for each $\alpha, t,\left\{\mathrm{r}_{4}(q, h, y, x)\right\}^{\alpha}(t) \simeq\{q\}(h,[y] *([\alpha(0) * x))$ and put $\mathrm{q}(e,[y+1] * x)=\left\langle 4, \mathrm{q}(e,[y] * x), \mathrm{r}_{4}(q, h, y, x)\right\rangle$.

Proof is as in Case S4. 
Case S6. Subcase (a). $\{e\}(\tau, x) \simeq\{g\}\left(\tau, x_{k+1}, x_{1}, \ldots, x_{k}, x_{k+2}, \ldots, x_{m}\right)$. As in Subcase (a) of Case S5, put $\mathrm{q}(e, x)=\left\langle 4,\langle 2,0\rangle, \mathrm{r}_{5}(q, g, e, x)\right\rangle$, where $\mathrm{r}_{5}(q, g, e, x)$ is primitive recursive and such that for each $\alpha, t$,

$$
\left\{\mathrm{r}_{5}(q, g, e, x)\right\}^{\alpha}(t) \simeq\{q\}\left(g,\left[x_{k+1}, x_{1}, \ldots, x_{k}, x_{k+2}, \ldots, x_{m}\right]\right) .
$$

Case S6. Subcase (b). $\{e\}(\tau, x) \simeq\{g\}\left(\tau_{k+1}, \tau_{1}, \ldots, \tau_{k}, \tau_{k+2}, \ldots, \tau_{n}, x\right)$. Choose a primitive recursive $\mathrm{r}_{6}(e)$ such that for each $\tau, t$,

$$
\left\{\mathrm{r}_{6}(e)\right\}^{\tau}(t)=\left\langle\tau_{k+1}, \tau_{1}, \ldots, \tau_{k}, \tau_{k+2}, \ldots, \tau_{n}\right\rangle(t)
$$

and put $\mathrm{q}(e, x)=\left\langle 6, \mathrm{q}(g, x), \mathrm{r}_{6}(e)\right\rangle$.

Case S7. $\{e\}(\tau, x)=\tau_{1}\left(x_{1}\right)$. Choose a primitive recursive $\mathrm{r}_{7}(x)$ such that for each $\alpha, t, \quad\left\{\mathrm{r}_{7}(x)\right\}^{\alpha}(t)=\left\langle 1,\left(\alpha\left((x)_{0}-1\right)\right)_{0}\right\rangle$ and put $\mathrm{q}(e, x)=\left\langle 4,\langle 2,0\rangle, \mathrm{r}_{7}(x)\right\rangle$. Then $\mathrm{f}^{\tau}(\mathrm{q}(e, x) ; t)=\mathrm{f}^{\tau}\left(\left\langle 1,\left(\tau\left(x_{1}\right)\right)_{0}\right\rangle ; t\right)=\left(\tau\left(x_{1}\right)\right)_{0}=\tau_{1}\left(x_{1}\right)$.

Case S8. Subcase (a). $\{e\}(\tau, x) \simeq \tau^{2}(\lambda u\{g\}(\tau, u, x))$. Choose a primitive recursive $\mathrm{r}_{8}(q, g, x)$ such that for each $\alpha, u,\left\{\mathrm{r}_{8}(q, g, x)\right\}^{\alpha}(u) \simeq\{q\}(g,[u] * x)$ and put $\mathrm{q}(e, x)=$ $\left\langle 3,\left\langle 4,\langle 2,0\rangle, \mathrm{r}_{8}(q, g, x)\right\rangle\right\rangle$.

If $\{e\}(\tau, x) \simeq w$, then by induction hypothesis $\mathrm{q}(g,[u] * x) \in N(\tau)$ for each $u$, hence $\left\langle 4,\langle 2,0\rangle, \mathrm{r}_{8}(q, g, x)\right\rangle \in N(\tau)$ and $\mathrm{f}^{\tau}\left(\left\langle 4,\langle 2,0\rangle, \mathrm{r}_{8}(q, g, x)\right\rangle ; u\right)=\{g\}(\tau, u, x)$. Thus $\mathrm{q}(e, x) \in N(\tau)$ and $\mathrm{f}^{\tau}(\mathrm{q}(e, x) ; t)=\tau^{2}\left(\lambda u \mathrm{f}^{\tau}\left(\left\langle 4,\langle 2,0\rangle, \mathrm{r}_{8}(q, g, x)\right\rangle ; u\right)\right)=\tau^{2}(\lambda u\{g\}(\tau, u, x))$.

Case S8. Subcase (b). $\{e\}(\tau, x) \simeq \tau^{3}(\lambda \alpha\{g\}(\alpha, \tau, x))$. Choose a primitive recursive $\mathrm{r}_{9}(e)$ such that for each $\alpha, \tau, t,\left\{\mathrm{r}_{9}(e)\right\}^{\alpha, \tau}(t) \simeq\langle\alpha(t)\rangle * \tau(t)$ and put

$$
\mathrm{q}(e, x)=\left\langle 5, \mathrm{q}(g, x), \mathrm{r}_{9}(e)\right\rangle \text {. }
$$

If $\{e\}(\tau, x) \simeq w$, then by induction hypothesis, for each $\alpha$,

$$
\mathrm{q}(g, x) \in N\left(\left\langle\alpha, \tau_{1}, \ldots, \tau_{n}\right\rangle\right)=N\left(\left\{\mathrm{r}_{9}(e)\right\}(\alpha, \tau)\right) ;
$$

hence $\mathrm{q}(e, x) \in N(\tau)$ and

$$
\mathrm{f}^{\tau}(\mathrm{q}(e, x) ; t)=\tau^{3}\left(\lambda \alpha \mathrm{f}\left(\left\{\mathrm{r}_{9}(e)\right\}(\alpha, \tau), \mathrm{q}(g, x) ; t\right)\right)=\tau^{3}(\lambda \alpha\{g\}(\alpha, \tau, x)) .
$$

Case S9. $\{e\}\left(\tau, \beta_{1}, \ldots, \beta_{k}, a, x, y_{1}, \ldots, y_{j}\right) \simeq\{a\}(\tau, x)$. Choose a primitive recursive $\mathrm{r}_{10}(e)$ such that for each $\alpha, t,\left\{\mathrm{r}_{10}(e)\right\}^{\alpha}(t)=\left\langle(\alpha(t))_{0},(\alpha(t))_{1}, \ldots,(\alpha(t))_{n-1}\right\rangle$ and put $\mathrm{q}\left(e,\left[x_{1}, \ldots, x_{m}, a, y_{1}, \ldots, y_{j}\right]\right)=\left\langle 6, \mathrm{q}(a, x), \mathrm{r}_{10}(e)\right\rangle$.

If $\{e\}\left(\tau, \beta_{1}, \ldots, \beta_{k}, a, x, y_{1}, \ldots, y_{j}\right) \simeq w$, then by induction hypothesis $\mathrm{q}(a, x) \in N(\tau)$ and for each $t, \mathrm{f}^{\tau}(\mathrm{q}(a, x) ; t)=w$. Hence

$$
\mathrm{q}\left(e,\left[x_{1}, \ldots, x_{m}, a, y_{1}, \ldots, y_{j}\right]\right) \in N\left(\left\langle\tau_{1}, \ldots, \tau_{n}, \beta_{1}, \ldots, \beta_{k}\right\rangle\right)
$$

and

$$
\mathrm{f}\left(\left\langle\tau_{1}, \ldots, \tau_{n}, \beta_{1}, \ldots, \beta_{k}\right\rangle, \quad \mathrm{q}\left(e,\left[x_{1}, \ldots, x_{m}, a, y_{1}, \ldots, y_{j}\right]\right) ; t\right)=\mathrm{f}(\mathrm{q}(a, x) ; t)=w .
$$

Case S10. Otherwise. Put $\mathrm{q}(e, x)=0$.

The recursion theorem gives us a partial recursive $\mathrm{q}(e, x)$ with Gödel number $q$ that satisfies all the above clauses. It is obvious that $\mathrm{q}(e, x)$ is completely defined; that it is primitive recursive can be inferred from the nature of the clauses, which (once $q$ is chosen) give a nested course-of-values recursion for $\mathrm{q}(e, x)$. 
We have already indicated in each of the clauses how to prove by induction on the definition of $\{e\}(\tau, x) \simeq w$ the implication from left to right in (1), and (2).

To prove the implication from right to left in (1) by induction on the definition of $\mathrm{q}(e, x) \in N(\tau)$, we first remark that $0 \notin N(\tau)$; thus if $\mathrm{q}(e, x) \in N(\tau)$, then $e$ and $x$ must satisfy one of the case hypotheses for cases $\mathrm{S} 1-\mathrm{S} 9$. It is a routine exercise to verify in each case (using the induction hypothesis) that $\{e\}(\tau, \boldsymbol{x})$ must be defined. (Notice the remark after Subcase (a) of Case S5.)

COROLlaRy 3.1. If $\mathrm{g}(t)$ is recursive in $\tau^{3}, \tau^{2}, \tau$, then there is a $z \in N(\tau)$ such that for all $t, \mathrm{~g}(t)=\mathrm{f}_{z}^{\tau}(t)$.

Proof. Let $g$ be an index of $\mathrm{g}(t)$, i.e., for all $t, \mathrm{~g}(t)=\{g\}\left(\tau^{3}, \tau^{2}, \tau, t\right)$; then for each $t, \mathrm{q}(g,[t]) \in N(\langle\tau\rangle)$ and $\mathrm{f}(\langle\tau\rangle, \mathrm{q}(g,[t]) ; t)=\mathrm{g}(t)$. Choose $e$ so that for each $\alpha, t,\{e\}^{\alpha}(t)=\mathrm{q}(g,[t])$ and let $w=\langle 4,\langle 2,0\rangle, e\rangle$; then $w \in N(\langle\tau\rangle)$ and $\mathrm{f}(\langle\tau\rangle, w ; t)=$ $\mathrm{g}(t)$. Choose $m$ so that for each $\tau,[m]^{\tau}(t)=\langle\tau(t)\rangle$ and let $z=\langle 6, w, m\rangle$; then $z \in N(\tau)$ and $\mathrm{f}_{z}^{\tau}(t)=\mathrm{f}_{w}^{\langle\tau\rangle}(t)=\mathrm{g}(t)$.

3. The hyperanalytic hierarchy. Let $\mathfrak{b}$ be a list of objects of types 2 and $3\left({ }^{4}\right)$, let $\tau^{i}(i=2,3)$ be the contraction of all objects of type $i$ in $\mathfrak{b}\left(\tau^{i}=\lambda \alpha^{i-1} 1\right.$, if $\mathfrak{b}$ contains no objects of type $i$ ). Using LXVII of RFII we obtain primitive recursive functions $\mathrm{c}(e)$ and $\mathrm{c}^{-1}(e)$ so that:

$$
\begin{aligned}
\{e\}(\mathfrak{b}, \tau, x) & \simeq\{\mathrm{c}(e)\}\left(\tau^{3}, \tau^{2}, \tau, x\right), \\
\{e\}\left(\tau^{3}, \tau^{2}, \tau, x\right) & \simeq\left\{c^{-1}(e)\right\}(\mathfrak{b}, \tau, x) .
\end{aligned}
$$

Definition 3. Let $\mathfrak{b}, \tau^{3}, \tau^{2}$ be related as above, let $\tau=\tau_{1}, \ldots, \tau_{n}$, put

$$
\mathfrak{a}=\mathfrak{b}, \boldsymbol{\tau} .
$$

(1) $N(\mathfrak{a})=N\left(\tau^{3}, \tau^{2},\langle\tau\rangle\right)$.

(2) For $z \in N(\mathfrak{a}), \mathrm{f}_{z}^{\mathfrak{a}}(t)=\mathrm{f}^{\mathfrak{a}}(z ; t)=\mathrm{f}(\mathfrak{a}, z ; t)=\mathrm{f}\left(\tau^{3}, \tau^{2},\langle\tau\rangle, z ; t\right)$.

(3) $\mathrm{r}(z)=\mathrm{c}^{-1}(\mathrm{p}(z))$, where $\mathrm{p}(z)$ is the function of Theorem 2 .

(4) $\mathrm{s}(e, \boldsymbol{x})=\mathrm{q}(\mathrm{c}(e),[x])$, where $\mathrm{q}(e, x)$ is the function of Theorem 3.

(5) For $z \in N(\mathfrak{a}),|z|^{\mathfrak{a}}=|z|(\mathfrak{a})=|z|^{\langle\tau\rangle}$.

(6) $\kappa(\mathfrak{a})=$ supremum $\left\{|z|^{\mathfrak{a}}: z \in N(\mathfrak{a})\right\}$.

(Recall that if $\tau$ is empty, then $\langle\tau\rangle=\langle\rangle=\lambda t 1$ and if $x$ is empty, $[x]=[]=1$; the clauses of Definition 3 apply to the cases of empty $\tau$ or $x$ with these conventions.)

(4) We collect here several notation conventions to which we adhere throughout the paper:

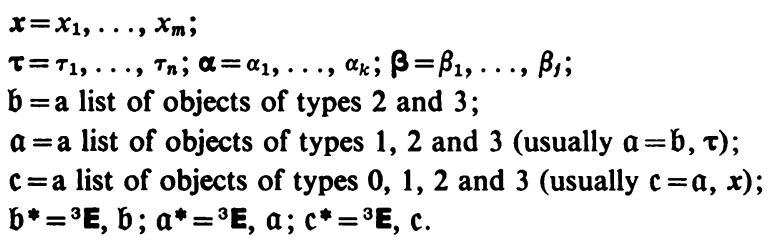


Using Theorems 2 and 3 we can easily verify the following results:

(A) $\{e\}(a, x) \downarrow \equiv \mathrm{s}(e, \boldsymbol{x}) \in N(\mathfrak{a})$.

(B) If $z \in N(\mathfrak{a})$, then $\lambda t\{\mathrm{r}(z)\}(\mathfrak{a}, t)$ is completely defined and $\mathrm{f}_{z}^{\mathfrak{a}}(t)=\{\mathrm{r}(z)\}(\mathfrak{a}, t)$.

(C) If $\mathrm{g}(t)$ is recursive in $\mathfrak{a}$, then there exists a $z \in N(\mathfrak{a})$ such that $\mathrm{g}(t)=\mathrm{f}_{z}^{\mathrm{a}}(t)$.

(D) If $(\alpha)[z \in N(\mathfrak{a}, \alpha)]$, then $\lambda \alpha t\{\mathrm{r}(z)\}(\mathfrak{a}, \alpha, t)$ is completely defined and

$$
\mathrm{f}(\mathfrak{a}, \alpha, z ; t)=\{\mathrm{r}(z)\}(\mathfrak{a}, \alpha, t) .
$$

(E) If $\mathrm{g}(a, t)$ is recursive in $\mathfrak{a}$, then there exists a $z$ such $(\alpha)[z \in N(\mathfrak{a}, \alpha)]$ and $\mathrm{g}(\alpha, t)=\mathrm{f}(\mathfrak{a}, \alpha, z ; t)$.

This "construction" of all functions and functionals recursive in $a$ by (transfinite) induction naturally defines a certain "hierarchy" on the one- and two-sections of $a$. However, if no assumptions are imposed on $\mathfrak{a}$, then this hierarchy may be trivial; e.g., if $a$ consists of recursive functions and functionals, then each function recursive in $\mathfrak{a}$ is recursive, and hence equal to some $\mathrm{f}_{z}^{\mathfrak{a}}(t)$ with $|z|^{\mathfrak{a}}=1$.

Put

$$
\mathfrak{b}^{*}={ }^{3} \mathbf{E}, \mathfrak{b}, \quad \mathfrak{a}^{*}={ }^{3} \mathbf{E}, \mathfrak{a}={ }^{3} \mathbf{E}, \mathfrak{b}, \boldsymbol{\tau}
$$

Relativizing Kleene's definition, we call a predicate, set or function hyperanalytic in $\mathfrak{a}$ if it is recursive in $\mathfrak{a}^{*}$.

In this section we define two transfinite sequences of predicates, ${ }_{1} \mathbf{G}_{z}(u, v)$ and ${ }_{2} \mathbf{G}_{z}(u, \alpha, v, \beta)\left(z \in N\left(\mathfrak{a}^{*}\right)\right)$ such that:

(1) ${ }_{i} \mathbf{G}_{z}$ is hyperanalytic in $\mathfrak{a}$, uniformly for $z \in N\left(\mathfrak{a}^{*}\right)$.

(2) If $|z|^{*} \leqq|w|^{a^{*}}$, then ${ }_{i} \mathbf{G}_{z}$ is recursive in ${ }_{i} \mathbf{G}_{w}$, uniformly for $z, w \in N\left(\mathfrak{a}^{*}\right)$.

(3) A function (functional) is hyperanalytic in a if and only if it is recursive in some ${ }_{1} \mathbf{G}_{z}\left({ }_{2} \mathbf{G}_{z}\right)$.

We also prove that the denumerable set of ordinals $|z|^{*}\left(z \in N\left(\mathfrak{a}^{*}\right)\right)$ has ordertype $\omega_{1}\left(\mathfrak{a}^{*}\right)=$ least denumerable ordinal which cannot be realized by an ordering hyperanalytic in $\mathfrak{a}$.

Lemma 3. Let $\mathfrak{b}$ be a list of objects of types 2 and 3 , let $\mathfrak{b}^{*}={ }^{3} \mathbf{E}, b$, let

$$
\boldsymbol{\alpha}=\alpha_{1}, \ldots, \alpha_{k}, \quad \boldsymbol{\beta}=\beta_{1}, \ldots, \beta_{j}
$$

be two lists of function variables. There is a primitive recursive $\mathrm{i}(k, j, z)$ such that, if $z \in N\left(\mathfrak{b}^{*}, \boldsymbol{\alpha}\right)$, then $\lambda w \boldsymbol{\beta}\{\mathrm{i}(k, j, z)\}\left(\mathfrak{b}^{*}, \boldsymbol{\alpha}, \boldsymbol{\beta}, w\right)$ is completely defined and

$$
|w|\left(\mathfrak{b}^{*}, \boldsymbol{\beta}\right) \leqq|z|\left(\mathfrak{b}^{*}, \boldsymbol{\alpha}\right) \equiv\{\mathrm{i}(k, j, z)\}\left(\mathfrak{b}^{*}, \boldsymbol{\alpha}, \boldsymbol{\beta}, w\right)=0 .
$$

(I.e. the initial segment of ordinals $|w|\left(\mathfrak{b}^{*}, \boldsymbol{\beta}\right)$ which are $\leqq|z|\left(\mathfrak{b}^{*}, \boldsymbol{\alpha}\right)$ is hyperanalytic in $\mathfrak{b}, \alpha$, uniformly for $z \in N\left(\mathfrak{b}^{*}, \boldsymbol{\alpha}\right)$.)

We do not prove this lemma here, since it will be an immediate consequence of Theorem 6. However one can easily construct an elementary proof similar to that of Theorem 2. 
LEMMA 4. Let $\mathfrak{a}=\mathfrak{b}, \tau$ be a list of objects of types $1,2,3$. The predicate $\lambda z z \in N(\mathfrak{a})$ is not recursive in $\mathfrak{a}$.

Proof. Suppose it were; put

$$
\begin{aligned}
\mathrm{g}(t) & =0 & & \text { if } t \notin N(\mathfrak{a}), \\
& =\mathrm{f}_{t}^{\mathfrak{a}}(t)+1 & & \text { if } t \in N(\mathfrak{a}) .
\end{aligned}
$$

Now (B) implies that $g(t)$ is recursive in $\mathfrak{a}$, though it is different from each $\mathrm{f}_{z}^{\mathfrak{a}}(t)$, contradicting (C).

Definition 4. Let $c$ be a list of variables of types $\leqq 3$. A predicate $P(\mathfrak{c})$ is recursively enumerable in $\mathfrak{a}($ r.e. in $\mathfrak{a})$, if for some $e$,

$$
P(\mathfrak{c}) \equiv\{e\}(\mathfrak{a}, \mathfrak{c}) \downarrow \text {. }
$$

LEMMA 5 (BOUNDEDNESS). If a predicate $P(\alpha, x)$ (with variables of types $\leqq 1$ ) is r.e. in $\mathfrak{a}^{*}$, then there exists a primitive recursive $\mathrm{g}(\boldsymbol{x})$ such that

$$
P(\boldsymbol{\alpha}, \boldsymbol{x}) \equiv \mathrm{g}(\boldsymbol{x}) \in N\left(\mathfrak{a}^{*}, \boldsymbol{\alpha}\right) .
$$

Moreover, if (1) holds with some $\mathrm{g}(\boldsymbol{x})$, hyperanalytic in $\mathfrak{a}$, then $P(\boldsymbol{\alpha}, \boldsymbol{x})$ is hyperanalytic in $a$ if and only if

$$
\text { supremum }\left\{|\mathrm{g}(\boldsymbol{x})|\left(\mathfrak{a}^{*}, \boldsymbol{\alpha}\right): P\left(\mathfrak{a}^{*}, \boldsymbol{\alpha}\right)\right\}<\kappa\left(\mathfrak{a}^{*}\right) .
$$

RemarK. We prove in Corollary 7.1 that the predicate $\lambda z \alpha z \in N\left(\mathfrak{a}^{*}, \boldsymbol{\alpha}\right)$ is r.e. in $\mathfrak{a}^{*}$.

Proof. For the first assertion, choose $e$ so that

$$
P(\boldsymbol{\alpha}, \boldsymbol{x}) \equiv\{e\}\left(\mathfrak{a}^{*}, \boldsymbol{\alpha}, \boldsymbol{x}\right) \downarrow
$$

and put $\mathrm{g}(\boldsymbol{x})=\mathrm{s}(e, \boldsymbol{x})$.

Assume that (1) and (2) are satisfied with a $g(x)$ hypernalytic in $\mathfrak{a}$; then for some $z \in N\left(\mathfrak{a}^{*}\right)$ we have

$$
\text { supremum }\left\{|\mathrm{g}(\boldsymbol{x})|\left(\mathfrak{a}^{*}, \boldsymbol{\alpha}\right): P(\boldsymbol{\alpha}, \boldsymbol{x})\right\} \leqq|z|\left(\mathfrak{a}^{*}\right)<\kappa\left(\mathfrak{a}^{*}\right) .
$$

Letting $\mathfrak{a}^{*}=\mathfrak{b}^{*}, \tau$ as usual $\left({ }^{4}\right)$, we then have

$$
\begin{aligned}
P(\boldsymbol{\alpha}, \boldsymbol{x}) & \equiv|\mathrm{g}(\boldsymbol{x})|\left(\mathfrak{a}^{*}, \boldsymbol{\alpha}\right) \leqq|z|\left(\mathfrak{a}^{*}\right) \\
& \equiv\{\mathrm{i}(n, n+k, z)\}\left(\mathfrak{b}^{*}, \tau, \tau, \alpha, g(x)\right)=0,
\end{aligned}
$$

so $P(\boldsymbol{\alpha}, \boldsymbol{x})$ is hypernalytic in $\mathfrak{a}$.

Now assume that (1) is satisfied with a $g(x)$ which is hyperanalytic in $\mathfrak{a}$, but

Then

$$
\text { supremum }\left\{|\mathrm{g}(\boldsymbol{x})|\left(\mathfrak{a}^{*}, \boldsymbol{\alpha}\right): P(\boldsymbol{\alpha}, \boldsymbol{x})\right\} \geqq \kappa\left(\mathfrak{a}^{*}\right) \text {. }
$$

$$
\begin{aligned}
z \in N\left(\mathfrak{a}^{*}\right) & \equiv(E \boldsymbol{\alpha})(E \boldsymbol{x})\left[P(\boldsymbol{\alpha}, \boldsymbol{x}) \&|z|\left(\mathfrak{a}^{*}\right) \leqq|\mathrm{g}(\boldsymbol{x})|\left(\mathfrak{a}^{*}, \boldsymbol{\alpha}\right)\right] \\
& \equiv(E \boldsymbol{\alpha})(E \boldsymbol{x})\left[P(\boldsymbol{\alpha}, \boldsymbol{x}) \&\left\{\mathrm{i}(n+k, n, \mathrm{~g}(\boldsymbol{x})\}\left(\mathfrak{b}^{*}, \boldsymbol{\tau} \boldsymbol{\alpha}, \boldsymbol{\tau}, z\right)=0\right]\right.
\end{aligned}
$$

If the predicate $P(\alpha, x)$ is hyperanalytic in $\mathfrak{a}$, the predicate in brackets is hyperanalytic in $\mathfrak{a}$; since the class of predicates hyperanalytic in $\mathfrak{a}$ is closed under number and function quantification, $z \in N\left(\mathfrak{a}^{*}\right)$ is hypernalytic in $\mathfrak{a}$, contradicting Lemma 4. 
DefinItION 5. For each $z \in N\left(\mathfrak{a}^{*}\right)\left({ }^{4}\right)$, put

$$
\begin{aligned}
{ }_{1} \mathbf{G}_{z}(u, v) & \equiv|v|^{\alpha^{*}} \leqq|z|^{a^{*}} \&|u|^{a^{*}} \leqq|v|^{\alpha^{*}}, \\
{ }_{2} \mathbf{G}_{z}(u, \alpha, v, \beta) & \equiv|v|^{a^{*}, \beta} \leqq|z|^{\alpha^{*}} \&|u|^{\alpha^{*}, \alpha} \leqq|v|^{\alpha^{*}, \beta} .
\end{aligned}
$$

THEOREM 4. (1) Each ${ }_{i} \mathbf{G}_{z}$ is hyperanalytic in $\mathfrak{a}$, uniformly for $z \in N\left(\mathfrak{a}^{*}\right)(i=1,2)$.

(2) If $|z|^{\mathfrak{a}^{*}}=|w|^{\alpha^{*}}$, then ${ }_{i} \mathbf{G}_{z} \equiv{ }_{i} \mathbf{G}_{w}(i=1,2)$.

(3) If $|z|^{a^{*}} \leqq|w|^{a^{*}}$, then ${ }_{i} \mathbf{G}_{z}$ is recursive in ${ }_{i} \mathbf{G}_{w}$, uniformly for $z, w \in N\left(\mathfrak{a}^{*}\right)$.

(4) $)_{1}$ If $P(x)$ is hyperanalytic in $\mathfrak{a}$, then $P(x)$ is recursive in some ${ }_{1} \mathbf{G}_{z}$.

$(4)_{2}$ If $P(\alpha, x)$ is hyperanalytic in $\mathfrak{a}$, then $P(\alpha, x)$ is recursive in some ${ }_{2} \mathbf{G}_{z}$.

Proof. (1) is an immediate consequence of Lemma 3 and (2) is obvious. To prove (3), notice that if $|z|^{a^{*}} \leqq|w|^{a^{*}}$, then

$$
{ }_{1} \mathbf{G}_{z}(u, v) \equiv{ }_{1} \mathbf{G}_{w}(v, z) \&{ }_{1} \mathbf{G}_{w}(u, v),
$$

and similarly for ${ }_{2} \mathbf{G}_{z},{ }_{2} \mathbf{G}_{w}$.

To prove $(4)_{2}$, choose a predicate $Q(\alpha, x)$ of one function variable with the same degree as $P(\alpha, x)$, e.g., $Q(\alpha, x) \equiv P\left((\alpha)_{1}, \ldots,(\alpha)_{k}, x\right)$. Now take $e$ so that

$$
Q(\alpha, \boldsymbol{x}) \equiv\{e\}\left(\mathfrak{a}^{*}, \alpha, \boldsymbol{x}\right) \downarrow \equiv s(e, \boldsymbol{x}) \in N\left(\mathfrak{a}^{*}, \alpha\right) .
$$

Since $Q(\alpha, x)$ is hyperanalytic, the Boundedness Lemma implies that for some $z$,

$$
\text { supremum }\left\{|\mathrm{s}(e, \boldsymbol{x})|\left(\mathfrak{a}^{*}, \alpha\right): Q(\alpha, \boldsymbol{x})\right\} \leqq|z|\left(\mathfrak{a}^{*}\right) \text {. }
$$

Thus $Q(\alpha, x) \equiv|\mathrm{s}(e, \boldsymbol{x})|\left(\mathfrak{a}^{*}, \alpha\right) \leqq|z|\left(\mathfrak{a}^{*}\right) \equiv{ }_{2} \mathbf{G}_{z}(\mathrm{~s}(e, \boldsymbol{x}), \alpha, \mathrm{s}(e, \boldsymbol{x}), \alpha)$.

Proof of $(4)_{1}$ is similar.

Definition 6. For each $\mathfrak{a}=\mathfrak{b}, \tau,\left\{|z|^{\mathfrak{a}}: z \in N(\mathfrak{a})\right\}$ is a countable set of ordinals; let $\psi$ be the unique order-preserving function which maps $\left\{|z|^{\mathfrak{a}}: z \in N(\mathfrak{a})\right\}$ onto an initial segment of the countable ordinals.

(1) $|z|_{\mathfrak{c}}^{\mathfrak{a}}=|z|_{\mathrm{c}}(\mathfrak{a})=\psi\left(|z|^{\mathfrak{a}}\right)(z \in N(\mathfrak{a}))$.

(2) $\omega_{1}(\mathfrak{a})=$ supremum $\left\{|z|_{\mathfrak{c}}^{\mathfrak{a}}: z \in N(\mathfrak{a})\right\}$.

(3) A countable ordinal $\eta$ is recursive in a, if there exists a function $\mathrm{g}(x, y)$, recursive in $\mathfrak{a}$, such that the relation $\lambda x y \mathrm{~g}(x, y)=0$ is a well-ordering of order-type $\eta$.

REMARK. For $z, w \in N(\mathfrak{a}),|z|^{\mathfrak{a}} \leqq|w|^{\mathfrak{a}} \equiv|z|_{\mathfrak{c}}^{\mathfrak{a}} \leqq|w|_{\mathfrak{a}}^{\mathfrak{a}}$.

THEOREM 5. $\omega_{1}\left(\mathfrak{a}^{*}\right)$ is the smallest countable ordinal which is not recursive in $\mathfrak{a}^{*}(\mathbf{4})$.

Proof. We first show that each $|z|_{c}^{a^{*}}$ is recursive in $\mathfrak{a}^{*}$. Put

$$
\begin{aligned}
& u \in C_{z} \equiv|u|^{\alpha^{*}} \leqq|z|^{a^{*}} \&(v)\left[|v|^{\alpha^{*}}=|u|^{\alpha^{*}} \rightarrow u \leqq v\right] \\
& \mathrm{g}(x, y)=0 \text { if } x \in C_{z} \& y \in C_{z} \&|x|^{a^{*}} \leqq|y|^{a^{*}} \\
&=1 \text { otherwise. }
\end{aligned}
$$

Lemma 3 implies that $\mathrm{g}(x, y)$ is recursive in $\mathrm{a}^{*}$, and it is obvious that $\lambda x y \mathrm{~g}(x, y)$ $=0$ is a well-ordering with order-type $|z|_{\mathrm{c}}^{a^{*}}$. 
To prove the converse, let $\preceq$ be a well-ordering recursive in $\mathfrak{a}^{*}$, with order-type $\eta$, put

$$
\begin{gathered}
D=\{\langle n, x\rangle: x \preceq x\} \cup\{5\}, \\
\mathrm{g}(x, y)=0 \quad \text { if } x \in D \& y \in D \\
\&\left[y=5 \vee\left[(x)_{1} \preceq(y)_{1}\right] \vee\left[(x)_{1}=(y)_{1} \&(x)_{0} \leqq(y)_{0}\right]\right], \\
=1 \text { otherwise. }
\end{gathered}
$$

It is clear that $\mathrm{g}(x, y)$ is recursive in $\mathfrak{a}^{*}$ and that $\lambda x y \mathrm{~g}(x, y)=0$ is a well-ordering with order-type $\eta \omega+1$. If $x_{0}$ is the minimum of the ordering $\preceq$, then the ordering on $D$ has the following properties:

(1) $\left\langle 0, x_{0}\right\rangle=3^{x_{0}}$ is the minimum.

(2) 5 is the maximum.

(3) The successor of any element $y \in D(y \neq 5)$ is $2 y$.

(4) An element of $D$ is a limit point if and only if it is 5 or of the form $3^{x}\left(x \neq x_{0}\right)$.

Let $z \in N\left(\mathfrak{a}^{*}\right)$ be such that

$$
\mathrm{f}_{z}^{*}(x)=\mathrm{g}\left((x)_{0},(x)_{1}\right),
$$

and choose a primitive recursive $\mathrm{m}(e, x)$ such that for each $\alpha$,

$$
\begin{aligned}
\{\mathrm{m}(e, x)\}^{\alpha}(t) & \simeq\langle 2,0\rangle & \text { if } \alpha(\langle t, x\rangle) \neq 0, \\
& \simeq\{e\}(t) & \text { if } \alpha(\langle t, x\rangle)=0 .
\end{aligned}
$$

We define a primitive recursive function $\mathrm{h}(u)$ from a Gödel number $h$ using the recursion theorem by:

$$
\begin{aligned}
\mathrm{h}(u) & \simeq\langle 2,0\rangle & & \text { if } u=\left\langle 0, x_{0}\right\rangle, \\
& \simeq\langle 3, \mathrm{~h}(x)\rangle & & \text { if } u=2 x \text { for some } x, \\
& \simeq\langle 4, z, \mathrm{~m}(h, u)\rangle & & \text { if } u=3^{x} \text { for some } x \neq x_{0}, \text { or } u=5, \\
& \simeq 0 & & \text { otherwise. }
\end{aligned}
$$

It is now easy to verify by transfinite induction on the ordering on $D$ that

$$
\begin{aligned}
u \in D \rightarrow \mathrm{h}(u) \in N\left(\mathrm{a}^{*}\right), & \\
u, v \in D \& \mathrm{~g}(u, v)=0 & \rightarrow|\mathrm{h}(u)|^{a^{*}} \leqq|\mathrm{~h}(v)|_{a^{*}} \\
& \rightarrow|\mathrm{h}(u)|_{c^{*}}^{a^{*}} \leqq|\mathrm{~h}(v)|_{c^{*}} .
\end{aligned}
$$

Thus $\mathrm{h}(5) \in N\left(\mathfrak{a}^{*}\right)$ and $\eta<\eta \omega+1 \leqq|\mathrm{~h}(5)|_{\mathrm{c}}{ }^{*}<\omega_{1}\left(\mathfrak{a}^{*}\right)$.

REMARK. Theorem 5 allows us to think of $N\left(a^{*}\right)$ as a notation system for the ordinals recursive in $\mathfrak{a}^{*}$, much as Kleene's $O$ is a notation system for the recursive ordinals.

For $i=1,2$, the predicates ${ }_{i} \mathbf{G}_{z}\left(z \in N\left(\mathfrak{a}^{*}\right)\right)$ provide a nondecreasing, transfinite sequence of degrees, indexed by the notations in $N\left(a^{*}\right)$, which exhausts the degrees in the $i$-section of $\mathfrak{a}^{*}$. One can easily see that this sequence need not be strictly increasing; however we shall prove in Corollary 8.1 that the sequence of degrees of the predicates $\mathbf{G}_{z}$ has order-type $\omega_{1}\left(\mathfrak{a}^{*}\right)$. 
REMARK. At the suggestion of the referee we outline a proof that for each $\mathfrak{a}, \kappa\left(\mathfrak{a}^{*}\right)>\aleph_{1}$. Using the notation of $\S 2$, consider the functional

$$
\begin{aligned}
\mathrm{h}(\xi, x, z, \tau, t) & =\mathrm{f}_{z}^{\tau}(t)+1 & & \text { if } \xi \in \mathrm{WO} \& x \in D_{\xi} \&|z|^{\tau} \leqq|x|_{\xi}, \\
& =0 & & \text { otherwise, }
\end{aligned}
$$

where for $x \in D_{\xi},|x|_{\xi}$ is the order-type of the initial segment of $\leqq_{\xi}$ up to and including $x$. It is easy to show by the methods of $\$ 1$ that the functional $h$ is hyperanalytic (in the fixed objects $\tau^{3}, \tau^{2}$ ). Now if each $|z|^{\tau}$ were countable, we would have

$$
z \in N(\tau) \equiv(E \xi)(E x)[\mathrm{h}(\xi, x, z, \tau, 0)>0] .
$$

In the notation of $\S 3$ this means that $z \in N\left(\mathfrak{a}^{*}\right)$ would be recursive in $\mathfrak{a}^{*}$, contradicting Lemma 4.

4. Minimum functions. In this section we prove two theorems on ordinal notations which are basic for the theory of predicates r.e. in $\mathfrak{a}^{*}$. The results are inspired by [2], where Gandy announces similar results for type 2.

We revert to the notation of $\S 2$, where $N(\tau), \mathrm{f}_{z}^{\tau}(t)$ and $|z|^{\tau}(z \in N(\tau))$ are defined, relative to arbitrary but fixed parameters $\tau^{2}, \tau^{3}$.

If $z \notin N(\tau)$, put $|z|^{\tau}=\operatorname{supremum}\left\{|w|^{\alpha}: w \in N(\alpha)\right\}$.

THEOREM 6. There is a partial recursive functional $\phi\left({ }^{3} \mathbf{E}, \tau^{3}, \tau^{2}, z, \alpha, w, \beta\right)$ $\simeq \phi(z, \alpha, w, \beta)$ such that:

(1) $\left[z \in N(\alpha) \&|z|^{\alpha} \leqq|w|^{\beta}\right] \rightarrow \phi(z, \alpha, w, \beta) \simeq 0$.

(2) $|w|^{\beta}<|z|^{\alpha} \rightarrow \phi(z, \alpha, w, \beta) \simeq 1$.

REMARK. Using Theorem 4, one can easily define a functional $\phi_{1}(z, \alpha, w, \beta)$ which will have properties (1) and (2) whenever $z \in N(\alpha)$, or a functional $\phi_{2}(z, \alpha, w, \beta)$ which will have properties (1) and (2) whenever $w \in N(\alpha)$. The nontrivial applications of Theorem 6 involve computing $\phi(z, \alpha, w, \beta)$ when we know $z \in N(\alpha) \vee w$ $\in N(\alpha)$, but do not know which of the disjuncts is true; in such cases $\phi(z, \alpha, w, \beta)$ gives us this information.

Proof. We define $\phi(z, \alpha, w, \beta)$ from its index $\phi$ using the recursion theorem.

For each number $u$ we consider seven cases $1-7$, according as $u$ is in one the forms $\langle 1, q\rangle,\langle 2,0\rangle,\left\langle 3, x_{1}\right\rangle,\left\langle 4, x_{1}, e\right\rangle,\left\langle 5, x_{1}, e\right\rangle,\left\langle 6, x_{1}, e\right\rangle$ (cases 1-6), or in none of these forms (case 7). In the computation we distinguish $7^{2}=49$ cases labeled $i j$ $(1 \leqq i \leqq 7,1 \leqq j \leqq 7)$, where in case $i j$ we assume that $z$ is in case $i$ and $w$ is in case $j$. (The computation is trivial, except when $3 \leqq i \leqq 6,3 \leqq j \leqq 6$.) For each of these cases we give informal instructions for computing $\phi(z, \alpha, w, \beta)$ in the manner of the proof of Theorem 1.

We shall be referring to the function $\mathrm{p}(z)$ of Theorem 2; recall that when $z \in N(\tau)$, then $\{\mathrm{p}(z)\}\left(\tau^{3}, \tau^{2}, \tau, t\right) \simeq \mathrm{f}_{z}^{\tau}(t)$.

Several times below we use the expression "ask ${ }^{2} \mathbf{E}$." In these instances we wish to emphasize that the predicate whose value we are determining only involves 
number quantification; surely questions to ${ }^{2} \mathbf{E}$ can be rephrased as questions to ${ }^{3} \mathbf{E}$.

A. Trivial cases.

A1. $1 \leqq i \leqq 2 \vee j=7$. Give (output) 0 .

A2. $[i=7 \& j<7] \vee[i \geqq 3 \& 1 \leqq j \leqq 2]$. Give 1 .

B. Cases $i j$ with $3 \leqq i \leqq 5,3 \leqq j \leqq 5$.

Case 33. $\left[z=\left\langle 3, z_{1}\right\rangle \& w=\left\langle 3, w_{1}\right\rangle\right]$. Give output $\phi\left(z_{1}, \alpha, w_{1}, \beta\right)$.

Case 34. $\left[z=\left\langle 3, z_{1}\right\rangle \& w=\left\langle 4, w_{1}, m\right\rangle\right]$.

?1. Compute $\phi\left(z_{1}, \alpha, w_{1}, \beta\right)$.

$11\left[\phi\left(z_{1}, \alpha, w_{1}, \beta\right) \simeq 0\right.$ in ?1]. Give 0.

?12 $\left[\phi\left(z_{1}, \alpha, w_{1}, \beta\right) \simeq 1\right.$ in ?1]. Let $\delta=\lambda u\left\{p\left(w_{1}\right)\right\}\left(\tau^{3}, \tau^{2}, \beta, u\right)$; ask ${ }^{2} \mathbf{E}$ if $\lambda s\{m\}^{\delta}(s)$ is completely defined.

121 [No to ?12]. Give 0.

?122 [Yes to ?12]. Ask ${ }^{2} E$ if $(E s)\left[\phi\left(z_{1}, \alpha,\{m\}^{\delta}(s), \beta\right)=0\right]$.

1221 [Yes to ?122]. Give 0.

1222 [No to ?122]. Give 1.

Case 43. $\left[z=\left\langle 4, z_{1}, e\right\rangle \& w=\left\langle 3, w_{1}\right\rangle\right]$. This case is symmetric to Case 34 . The computation is that of Case 34, except that $z$ and $w, z_{1}$ and $w_{1}, \alpha$ and $\beta$ and $e$ and $m$ are interchanged; however we do not change the order of the arguments in $\phi$. We give this symmetric computation here so we can omit it in some other cases later.

?1. Compute $\phi\left(z_{1}, \alpha, w_{1}, \beta\right)$.

$11\left[\phi\left(z_{1}, \alpha, w_{1}, \beta\right) \simeq 1\right.$ in ?1]. Give 1 .

?12 $\left[\phi\left(z_{1}, \alpha, w_{1}, \beta\right) \simeq 0\right.$ in ?1]. Let $\gamma=\lambda u\left\{\mathrm{p}\left(z_{1}\right)\right\}\left(\tau^{3}, \tau^{2}, \alpha, u\right)$; ask ${ }^{2} E$ if $\lambda t\{e\}^{\gamma}(t)$ is completely defined.

121 [No to ?12]. Give 1.

?122 [Yes to ?12]. Ask ${ }^{2} E$ if $(E t)\left[\phi\left(\{e\}^{\gamma}(t), \alpha, w_{1}, \beta\right)=1\right]$.

1221 [Yes to ?122]. Give 1.

1222 [No to ?122]. Give 0.

Case 35. $\left[z=\left\langle 3, z_{1}\right\rangle \& w=\left\langle 5, w_{1}, m\right\rangle\right]$.

?1. Ask ${ }^{3} E$ if $m$ defines a $\beta$-recursive functional $\{m\}(\delta, \beta)$.

11 [No to ?1]. Give 0.

?12 [Yes to ?1]. Ask ${ }^{3} E$ if $(E \delta)\left[\phi\left(z_{1}, \alpha, w_{1},\{m\}(\delta, \beta)\right)=0\right]$.

121 [Yes to ?12]. Give 0.

122 [No to ?12]. Give 1.

Case 53. $\left[z=\left\langle 5, z_{1}, e\right\rangle \& w=\left\langle 3, w_{1}\right\rangle\right]$. Symmetric to Case 35.

Case 44. $\left[z=\left\langle 4, z_{1}, e\right\rangle \& w=\left\langle 4, w_{1}, m\right\rangle\right]$.

?1. Compute $\phi\left(z_{1}, \alpha, w_{1}, \beta\right)$.

?11 $\left[\phi\left(z_{1}, \alpha, w_{1}, \beta\right) \simeq 0\right.$ in ?1]. Let $\gamma=\lambda u\left\{\mathrm{p}\left(z_{1}\right)\right\}\left(\tau^{3}, \tau^{2}, \alpha, u\right)$; ask ${ }^{2} E$ if $\lambda t\{e\}^{\gamma}(t)$ is completely defined.

111 [No to ?11]. Give 1.

?112 [Yes to ?11]. Ask ${ }^{2} E$ if $(t)\left[\phi\left(\{e\}^{\gamma}(t), \alpha, w_{1}, \beta\right)=0\right]$.

1121 [Yes to ?112]. Give 0. 
?1122 [No to ?112]. Let $\delta=\lambda u\left\{\mathrm{p}\left(w_{1}\right)\right\}\left(\tau^{3}, \tau^{2}, \beta, u\right)$; ask ${ }^{2} \mathrm{E}$ if $\lambda s\{m\}^{\delta}(s)$ is completely defined.

11221 [No to ?1122]. Give 0.

?11222 [Yes to ?1122]. Ask ${ }^{2}$ E if

$(t)\left[\phi\left(\{e\}^{\gamma}(t), \alpha, w_{1}, \beta\right)=0 \vee(E s)\left[\phi\left(\{e\}^{\gamma}(t), \alpha,\{m\}^{\delta}(s), \beta\right)=0\right]\right]$.

112221 [Yes to ?1122]. Give 0.

112222 [No to ?1122]. Give 1.

?12 [ $\phi\left(z_{1}, \alpha, w_{1}, \beta\right) \simeq 1$ in ?1]. (This subcase is symmetric to ?11, and the computation is symmetric to ?11-112222.) Let $\delta=\lambda u\left\{\mathrm{p}\left(w_{1}\right)\right\}\left(\tau^{3}, \tau^{2}, \beta, u\right)$; ask ${ }^{2} \mathbf{E}$ if $\lambda s\{m\}^{\delta}(s)$ is completely defined.

121 [No to ?12]. Give 0.

?122 [Yes to ?12]. Ask ${ }^{2} E$ if $(s)\left[\phi\left(z_{1}, \alpha,\{m\}^{\delta}(s), \beta\right)=1\right]$.

1221 [Yes to ?122]. Give 1.

?1222 [No to ?122]. Let $\gamma=\lambda u\left\{\mathrm{p}\left(z_{1}\right)\right\}\left(\tau^{3}, \tau^{2}, \alpha, u\right)$; ask ${ }^{2} \mathrm{E}$ if $\lambda t\{e\}^{\gamma}(t)$ is completely defined.

12221 [No to ?1222]. Give 1.

?12222 [Yes to ?1222]. Ask ${ }^{2} \mathbf{E}$ if

$(s)\left[\phi\left(z_{1}, \alpha,\{m\}^{\delta}(s), \beta\right)=1 \vee(E t)\left[\phi\left(\{e\}^{\gamma}(t), \alpha,\{m\}^{\delta}(s), \beta\right)=1\right]\right]$.

122221 [Yes to ?12222]. Give 1.

122222 [No to ?12222]. Give 0.

Case 45. $\left[z=\left\langle 4, z_{1}, e\right\rangle \& w=\left\langle 5, w_{1}, m\right\rangle\right]$.

?1. Ask ${ }^{3} E$ if $m$ defines a $\beta$-recursive functional $\{m\}(\delta, \beta)$.

11 [No to ?1]. Give 0.

?12 [Yes to ?1]. Ask ${ }^{3} E$ if $(E \delta)\left[\phi\left(z_{1}, \alpha, w_{1},\{m\}(\delta, \beta)\right)=0\right]$.

121 [No to ?12]. Give 1.

?122 [Yes to ?12]. Let $\gamma=\lambda u\left\{p\left(z_{1}\right)\right\}\left(\tau^{3}, \tau^{2}, \alpha, u\right)$; $\operatorname{ask}^{2} \mathbf{E}$ if $\lambda t\{e\}^{\gamma}(t)$ is completely defined.

1221 [No to ?122]. Give 1.

1222 [Yes to ?122]. Ask ${ }^{3} E$ if $(t)(E \delta)\left[\phi\left(\{e\}^{\gamma}(t), \alpha, w_{1},\{m\}(\delta, \beta)\right)=0\right]$.

12221 [Yes to ?1222]. Give 0.

12222 [No to ?1222]. Give 1.

Case 54. $\left[z=\left\langle 5, z_{1}, e\right\rangle \& w=\left\langle 4, w_{1}, m\right\rangle\right]$. Symmetric to Case 45.

Case 55. $\left[z=\left\langle 5, z_{1}, e\right\rangle \& w=\left\langle 5, w_{1}, m\right\rangle\right]$.

?1. Ask ${ }^{3} \mathbf{E}$ if $e$ defines an $\alpha$-recursive functional $\{e\}(\gamma, \alpha)$.

11 [No to ?1]. Give 1.

?12 [Yes to ?1]. Ask ${ }^{3} E$ if $m$ defines a $\beta$-recursive functional $\{m\}(\delta, \beta)$.

121 [No to ?12]. Give 0.

?122 [Yes to ?12]. Ask ${ }^{3} E$ if $(\gamma)(E \delta)\left[\phi\left(z_{1},\{e\}(\gamma, \alpha), w_{1},\{m\}(\delta, \beta)\right)=0\right]$.

1221 [Yes to ?122]. Give 0.

1222 [No to ?122]. Give 1.

C. Cases $i j$ with $i \geqq 3 \& j \geqq 3 \&[i=6 \vee j=6]$.

A number $w=\left\langle 6, w_{1}, m\right\rangle$ is a member of $N(\beta)$ if and only if $\lambda s\{m\}^{\beta}(s)=\delta$ is 
completely defined and $w_{1} \in N(\delta)$; and in that case $|w|^{\beta}=\left|w_{1}\right|^{\delta}+1$. Thus, once we verify that $\lambda s\{m\}^{\beta}(s)$ is completely defined, case $i 6$ is treated very much like case $i 3$, the other case where $|w|^{\beta}$ is always a successor ordinal (if $w \in N(\beta)$ ). We give one of these cases and leave the rest for the reader.

Case 36. $\left[z=\left\langle 3, z_{1}\right\rangle \& w=\left\langle 6, w_{1}, m\right\rangle\right]$.

?1. Ask ${ }^{2} \mathbf{E}$ if $\lambda s\{m\}^{\beta}(s)=\delta$ is completely defined.

11 [No to ?1]. Give 0.

?12 [Yes to ?1]. Compute $\phi\left(z_{1}, \alpha, w_{1}, \delta\right)$.

$121\left[\phi\left(z_{1}, \alpha, w_{1}, \delta\right) \simeq 0\right]$. Give 0.

$122\left[\phi\left(z_{1}, \alpha, w_{1}, \delta\right) \simeq 1\right]$. Give 1 .

To prove that this functional $\phi(z, \alpha, w, \beta)$ satisfies the conclusion of the theorem, we first show by induction on $z \in N(\alpha)$ that, if $|z|^{\alpha} \leqq|w|^{\beta}$, then $\phi(z, \alpha, w, \beta) \simeq 0$ and then by induction on $w \in N(\beta)$ that if $|w|^{\beta}<|z|^{\alpha}$, then $\phi(z, \alpha, w, \beta) \simeq 1$. The induction on $z \in N(\alpha)$ breaks down into 42 cases (since $z$ must be in one of cases 1-6 while $w$ is arbitrary) of which only 16 are nontrivial. Similarly the induction on $w \in N(\beta)$ has but 16 nontrivial cases.

Let us say that 0 is the proper value (for $z, \alpha, w, \beta)$ if $z \in N(\alpha) \&|z|^{\alpha} \leqq|w|^{\beta}$ and that 1 is the proper value (for $z, \alpha, w, \beta$ ) if $|w|^{\beta}<|z|^{\alpha}$ (which implies $w \in N(\beta)$ ). We must verify in each of the 16 nontrivial cases, and under each of the two assumptions, that 0 or 1 is the proper value, that the computation yields that proper value as output. Each time we may assume as induction hypothesis that the computation leads to the proper value for arguments that are "predecessors" of $z$ or $w$ as members of $N(\alpha)$ or $N(\beta)$.

In 33 the verification is immediate, since the ind. hyp. guarantees that the proper value for $z_{1}, \alpha, w_{1}, \beta$ is the same as the proper value for $z, \alpha, w, \beta$.

Consider 34 and assume that the proper value is 0 . We claim that the computation will be along one of the branches of Diagram A below and will reach the end of some branch, thus giving output 0 .

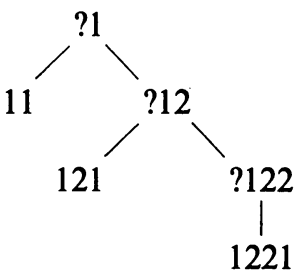

Diagram A.

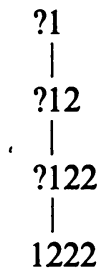

Diagram B.

Case $34 ;$ proper value $=0 . \quad$ Case $34 ;$ proper value $=1$.

To substantiate the claim we must verify that each time a question is asked in that computation, the partial predicate involved is defined and the answer leads the computation along one of the branches of the diagram. The ind. hyp. guarantees that $\phi\left(z_{1}, \alpha, w_{1}, \beta\right)$ is defined, hence ?1 is answered. If $\phi\left(z_{1}, \alpha, w_{1}, \beta\right) \simeq 1$ and we go 
to ?12, then by ind. hyp. we know that $\left|w_{1}\right|^{\beta}<\left|z_{1}\right|^{\alpha}$, hence $w_{1} \in N(\beta)$. Thus $\delta=\lambda u\left\{\mathrm{p}\left(w_{1}\right)\right\}\left(\tau^{3}, \tau^{2}, \beta, u\right)$ is completely defined and the Substitution Lemma (which is tacitly appealed to in ?12) guarantees that ?12 is answered. If the answer to ?12 leads us to ?122, then we know that $\lambda s\{m\}^{\delta}(s)$ is completely defined, and by ind. hyp. $\phi\left(z_{1}, \alpha,\{m\}^{\delta}(s), \beta\right)$ is defined for each $s$, so again $? 122$ is well posed for the Substitution Lemma. Now the ind. hyp. implies that the answer to ?122 must be "yes," since the assumption that it is "no" together with the ind. hyp. easily leads to the conclusion $|w|^{\beta}<|z|^{\alpha}$.

A similar verification is needed for each of the two assumptions, that 0 or $\mathbf{1}$ is the proper value, in each of the nontrivial cases. One simple way to obtain these verifications is to draw diagrams of all the possible computation paths and check that the proper value is reached along each allowable branch. We draw one more of these diagrams and omit the details.

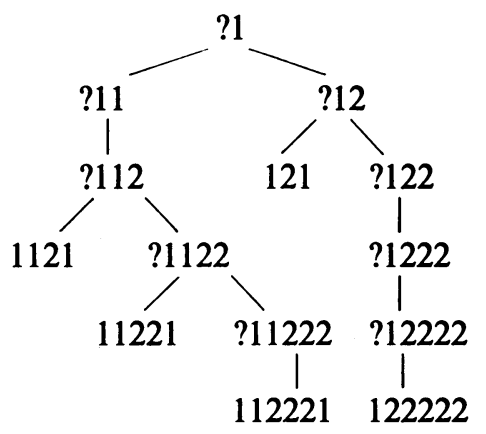

Diagram C.

Case 44; proper value $=0$.

THEOREM 7. There is a partial recursive $\psi\left({ }^{3} \mathbf{E}, \tau^{3}, \tau^{2}, \tau, h\right) \simeq \psi(\tau, h)$ such that $\lambda t\{h\}(t)$ is completely defined \& $(E t)[\{h\}(t) \in N(\tau)] \rightarrow(t) \mid\left(\left.\{h\}(\psi(\tau, h))\right|^{\tau} \leqq|\{h\}(t)|^{\tau}\right.$. (In particular,

$$
\lambda t\{h\}(t) \text { comp. defined \& }(E t)[\{h\}(t) \in N(\tau)] \rightarrow\{h\}(\psi(\tau, h)) \in N(\tau) .)
$$

Proof. We shall define $\psi(\tau ; h) \simeq \psi\left({ }^{3} \mathbf{E}, \tau^{3}, \tau^{2}, \tau, h\right)$ from an index $\psi$ by the recursion theorem in the usual way. We assume throughout that $\lambda t\{h\}(t)$ is completely defined.

If $(E t)[\{h\}(t) \in N(\tau)]$, put

$$
\operatorname{rank}(h)=\text { least } s(t)\left[|\{h\}(s)|^{\tau} \leqq|\{h\}(t)|^{\tau}\right] .
$$

Let $h^{\prime}$ be a primitive recursive function of $h$ such that

$$
\left\{h^{\prime}\right\}(t) \simeq\{h\}(t+1)
$$

We notice that if rank $(h)>0$, then $\operatorname{rank}\left(h^{\prime}\right)=\operatorname{rank}(h)-1$. 
Recall that by Theorem 3:

$$
\psi(\tau, h) \downarrow \equiv \mathrm{q}(\bar{\psi},[h]) \in N(\tau) .
$$

In the instructions for the computation of $\psi(\tau, h)$ from $\psi$ we write $\mathrm{h}(t)$ for $\{h\}(t)$.

?1. Compute $\phi\left(\mathrm{h}(0), \tau, \mathrm{q}\left(\bar{\psi},\left[h^{\prime}\right]\right), \tau\right)$.

?11 $\left[\phi\left(\mathrm{h}(0), \tau, \mathrm{q}\left(\bar{\psi},\left[h^{\prime}\right]\right), \tau\right) \simeq 0\right.$ in ?1]. Ask ${ }^{2} \mathbf{E}$ if $(t)[\phi(\mathrm{h}(0), \tau, \mathrm{h}(t), \tau)=0]$.

111 [Yes to ?11]. Give 0.

112 [No to ?11]. Give $\psi\left(\tau, h^{\prime}\right)+1$.

?12 $\left[\phi\left(\mathrm{h}(0), \tau, \mathrm{q}\left(\bar{\psi},\left[h^{\prime}\right]\right), \tau\right) \simeq 1\right.$ in ?1]. Compute $\phi\left(\mathrm{h}(0), \tau, \mathrm{h}\left(\psi\left(\tau, h^{\prime}\right)+1\right), \tau\right)$.

$121\left[\phi\left(\mathrm{h}(0), \tau, \mathrm{h}\left(\psi\left(\tau, h^{\prime}\right)+1\right), \tau\right) \simeq 0\right.$ in ?12]. Follow the instructions of ?11.

$122\left[\phi\left(\mathrm{h}(0), \tau, \mathrm{h}\left(\psi\left(\tau, h^{\prime}\right)+1\right), \tau\right) \simeq 1\right.$ in ?12]. Give $\psi\left(\tau, h^{\prime}\right)+1$.

We prove that if $(E t)[\mathrm{h}(t) \in N(\tau)]$, then $\psi(\tau, h)$ gives the correct output rank $(h)$, by induction on rank $(h)$.

BasIS. Rank $(h)=0$, i.e., $(t)\left[|\mathrm{h}(0)|^{\tau} \leqq|\mathrm{h}(t)|^{\tau}\right]$. Since $\mathrm{h}(0) \in N(\tau)$,

$$
\phi\left(\mathrm{h}(0), \tau, \mathrm{q}\left(\bar{\psi},\left[h^{\prime}\right]\right), \tau\right)
$$

is defined and ?1 is answered. If the answer sends us to ?11, the answer to this must be "yes," so by 111 the output is correctly 0 . If the answer to ?1 sends us to ?12, then $\left|\mathrm{q}\left(\bar{\psi},\left[h^{\prime}\right]\right)\right|^{\tau}<|\mathrm{h}(0)|^{\tau}$, so $\mathrm{q}\left(\bar{\psi},\left[h^{\prime}\right]\right) \in N(\tau)$, i.e., $\psi\left(\tau,\left[h^{\prime}\right]\right)$ is defined, so ?12 is answered; now the answer must lead us to 121 and thence to ?11 and the correct output, since by assumption $|\mathrm{h}(0)|^{\tau} \leqq\left|\mathrm{h}\left(\psi\left(\tau, h^{\prime}\right)+1\right)\right|^{\tau}$.

INDUCTION STEP. Rank $(h)>0$. Now we may assume that $\psi\left(\tau, h^{\prime}\right) \simeq \operatorname{rank}\left(h^{\prime}\right)$; we must show that $\psi(\tau, h) \simeq \psi\left(\tau, h^{\prime}\right)+1$. Since $\psi\left(\tau, h^{\prime}\right)$ is defined, $\mathrm{q}\left(\bar{\psi},\left[h^{\prime}\right]\right) \in N(\tau)$, so ?1 is answered. If the answer to ?1 leads us to ?11, the answer to this must be "no," so we are led to 112 and the correct output. If the answer to ?1 leads us to ?12, then from the answer to that we either go to ?11 and thence to the correct output as before or directly to the correct output through 122 .

5. Predicates r.e. in ${ }^{3} \mathbf{E}$.

Definition 7. Let $\mathfrak{b}, \tau^{3}, \tau^{2}, \tau$ and $\boldsymbol{x}$ be as in Definition 3 , let $\alpha=\alpha_{1}, \ldots, \alpha_{k}$, $\beta=\beta_{1}, \ldots, \beta_{j}$.

(1) $\phi(\mathfrak{b}, z, \boldsymbol{\alpha}, w, \boldsymbol{\beta}) \simeq \phi\left({ }^{3} \mathbf{E}, \tau^{3}, \tau^{2}, z,\langle\boldsymbol{\alpha}\rangle, w,\langle\boldsymbol{\beta}\rangle\right)$.

(2) $\psi(\mathfrak{b}, \tau, h) \simeq \psi\left({ }^{3} \mathbf{E}, \tau^{3}, \tau^{2},\langle\tau\rangle, h\right)$.

(3) Let $\mathrm{h}(e, x)$ be primitive recursive and such that

$$
\{\mathrm{h}(e, \boldsymbol{x})\}(t)=\mathrm{s}(e, \boldsymbol{x}, t),
$$

put

$$
\nu(\mathfrak{b}, \tau, \boldsymbol{x}, e) \simeq \psi(\mathfrak{b}, \tau, \mathrm{h}(e, \boldsymbol{x}))
$$

The functionals $\phi, \psi$ and $\nu$ are clearly partial recursive in ${ }^{3} \mathbf{E}$. In particular, if $\mathfrak{b}^{*}={ }^{3} \mathbf{E}, \mathfrak{b}$, then $\lambda z \alpha w \beta \phi\left(\mathfrak{b}^{*}, z, \boldsymbol{\alpha}, w, \boldsymbol{\beta}\right)$ is partial recursive in $\mathfrak{b}^{*}$, and similarly with $\psi$ and $\nu$. 
For any list $\mathfrak{a}=\mathfrak{b}, \tau\left({ }^{4}\right)$, if $z \notin N(\mathfrak{a})$, put $|z|^{\mathfrak{a}}=\operatorname{supremum}\left\{|z|^{\mathfrak{b}, \alpha}: w \in N(\mathfrak{b}, \boldsymbol{\alpha})\right\}$. Using Theorems 6 and 7 we can easily verify the following results:

(F) $z \in N(\mathfrak{b}, \boldsymbol{\alpha}) \&|z|(\mathfrak{b}, \boldsymbol{\alpha}) \leqq|w|(\mathfrak{b}, \boldsymbol{\beta}) \rightarrow \phi(\mathfrak{b}, z, \boldsymbol{\alpha}, w, \boldsymbol{\beta}) \simeq 0$.

(G) $|w|(\mathfrak{b}, \boldsymbol{\beta})<|z|(\mathfrak{b}, \boldsymbol{\alpha}) \rightarrow \phi(\mathfrak{b}, z, \boldsymbol{\alpha}, w, \boldsymbol{\beta}) \simeq 1$.

(H) Let $\mathfrak{a}=\mathfrak{b}, \tau, \mathrm{h}(t) \simeq\{h\}(t)$; then

$\lambda t \mathrm{~h}(t)$ comp. defined \& $(E t)\{\mathrm{h}(t) \in N(\mathfrak{a})] \rightarrow \psi(\mathfrak{a}, h) \downarrow \&(t)\left[|\mathrm{h}(\psi(\mathfrak{a}, h))|^{\mathfrak{a}} \leqq|\mathrm{h}(t)|^{\mathfrak{a}}\right]$.

(I) Let $\mathfrak{c}=\mathfrak{b}, \tau, \boldsymbol{x}$; then

$$
(E t)[\{e\}(\mathfrak{c}, t) \downarrow] \rightarrow \nu(\mathfrak{c}, e) \downarrow \&\{e\}(\mathfrak{c}, \nu(\mathfrak{c}, e)) \downarrow .
$$

From (F), with $\mathfrak{b}^{*}$ for $\mathfrak{b}$, we can easily prove Lemma 3; (I) is Gandy's main result in [2], for type 3.

These results allow us to establish several elementary properties of the class of predicates r.e. in ${ }^{3} \mathbf{E}$. (We leave two basic normal form theorems for §6.) Contrary to the situation in $\S 3$, where we had to restrict ourselves to predicates with variables of types $\leqq 1$, we study here predicates $P(\mathfrak{c})$, r.e. in ${ }^{3} \mathbf{E}$, where $c$ is a list of variables of types $\leqq 3$ (Definition 4). Relativized versions of Theorems 7 and 8 are obtained by substituting specific functionals for some of the variables in the list $c$.

(Added November 1966. We thank Abraham Robinson for showing to us in May, 1966 a mimeographed copy of R. Platek, Foundations of recursion theory, Ph.D. Thesis, Stanford University, 1966. This mimeographed copy (dated January, 1966) contains a proof of (I) (with a different $v$ ) as well as proofs of the immediate corollaries of (I) in Theorem 7 and Theorem 8 below. Platek's independent proofs of these results utilize an analysis of hyperanalytic computations that is based on a $\lambda$-calculus instead of our analysis through the induction in Definition 1.)

Theorem 7. (1) If $P(\mathfrak{c}, y)$ is r.e. in ${ }^{3} \mathbf{E}$, then $(E y) P(\mathfrak{c}, y)$ is also r.e. in ${ }^{3} \mathbf{E}$.

(2) If $P(\mathfrak{c})$ and $Q(\mathfrak{c})$ are r.e in ${ }^{3} \mathbf{E}$, then $P(\mathfrak{c}) \vee Q(\mathfrak{c})$ is also r.e. in ${ }^{3} \mathbf{E}$.

(3) A predicate $P(\mathfrak{c})$ is recursive in ${ }^{3} \mathbf{E}$ (hyperanalytic) if and only if both $P(\mathfrak{c})$ and $\bar{P}(\mathbf{c})$ are r.e. in ${ }^{3} \mathbf{E}$.

(4) Let $P(\mathfrak{c})$ and $Q(\mathfrak{c})$ be r.e. in ${ }^{3} \mathbf{E}$. There exist predicates $P_{1}(\mathfrak{c})$ and $Q_{1}(\mathfrak{c})$, r.e. in ${ }^{3}$ E. such that:

(a) $P_{1}($ c $) \rightarrow P($ c $) ; Q_{1}(\mathrm{c}) \rightarrow Q(\mathrm{c})$.

(b) $P(\mathrm{c}) \vee Q(\mathrm{c}) \rightarrow P_{1}(\mathrm{c}) \vee Q_{1}(\mathrm{c})$.

(c) It is impossible that $P_{1}(\mathrm{c}) \& Q_{1}(\mathrm{c})$.

Proof. (1) Put $\mathrm{c}^{*}={ }^{3} \mathrm{E}, \mathrm{c}$ and choose $e$ so that $P(\mathfrak{c}, y) \equiv\{e\}\left(\mathrm{c}^{*}, y\right) \downarrow$; now (I) implies that $(E y) P(\mathfrak{c}, y) \equiv\{e\}\left(\mathrm{c}^{*}, \nu\left(\mathrm{c}^{*}, e\right)\right) \downarrow$.

(2) follows immediately from (1).

(3) The "only if" part is trivial. To prove the "if" part, write $\mathfrak{c}=\mathfrak{b}, \tau, \boldsymbol{x}\left({ }^{4}\right)$, put $\mathfrak{b}^{*}={ }^{3} \mathbf{E}, \mathfrak{b}$, and choose $e, m$ so that

$$
\begin{aligned}
& P(\mathfrak{c}) \equiv\{e\}\left(\mathfrak{b}^{*}, \tau, x\right) \downarrow \equiv \mathrm{s}(e, x) \in N\left(\mathfrak{b}^{*}, \tau\right), \\
& \bar{P}(\mathfrak{c}) \equiv\{m\}\left(\mathfrak{b}^{*}, \tau, x\right) \downarrow \equiv \mathrm{s}(m, x) \in N\left(\mathfrak{b}^{*}, \tau\right) .
\end{aligned}
$$


Now for each $\mathrm{c}$, exactly one of $\mathrm{s}(e, \boldsymbol{x}), \mathrm{s}(m, \boldsymbol{x})$ is a member of $N\left(\mathfrak{b}^{*}, \tau\right)$; hence $\lambda c \phi\left(\mathfrak{b}^{*}, \mathrm{~s}(e, \boldsymbol{x}), \tau, \mathrm{s}(m, \boldsymbol{x}), \tau\right)$ is completely defined and

$$
P(\mathfrak{c}) \equiv \phi\left(\mathfrak{b}^{*}, \mathrm{~s}(e, \boldsymbol{x}), \tau, \mathrm{s}(m, \boldsymbol{x}), \tau\right)=0 .
$$

(4) As in (3), pick $e, m$ so that

Put

$$
\begin{aligned}
& P(\mathfrak{c}) \equiv \mathrm{s}(e, \boldsymbol{x}) \in N\left(\mathfrak{b}^{*}, \tau\right), \\
& Q(\mathfrak{c}) \equiv \mathrm{s}(m, \boldsymbol{x}) \in N\left(\mathfrak{b}^{*}, \tau\right) .
\end{aligned}
$$

$$
\begin{aligned}
& P_{1}(\mathfrak{c}) \equiv P(\mathfrak{c}) \& \phi\left(\mathfrak{b}^{*}, \mathrm{~s}(e, x), \tau, \mathrm{s}(m, x), \tau\right) \simeq 0, \\
& Q_{1}(\mathfrak{c}) \equiv Q(\mathfrak{c}) \& \phi\left(\mathfrak{b}^{*}, \mathrm{~s}(e, x), \tau, \mathrm{s}(m, x), \tau\right) \simeq 1 .
\end{aligned}
$$

The verification that $P_{1}$ and $Q_{1}$ have the desired properties is easy.

Corollary 7.1. Let $\mathfrak{a}=\mathfrak{b}, \tau, \mathfrak{a}^{*}={ }^{3} \mathbf{E}$, a. The predicate $\lambda z \mathfrak{a} z \in N\left(\mathfrak{a}^{*}\right)$ is r.e. in ${ }^{3} \mathbf{E}$.

Proof. By (A),

$$
\{e\}\left(\mathfrak{a}^{*}, x\right) \downarrow \equiv \mathrm{s}(e, x) \in N\left(\mathfrak{a}^{*}\right)
$$

We claim that for each $\mathfrak{a}$,

$$
\text { supremum }\left\{|\mathrm{s}(e, x)|^{\mathfrak{a}^{*}}:\{e\}\left(\mathfrak{a}^{*}, x\right) \downarrow\right\}=\kappa\left(\mathfrak{a}^{*}\right) ;
$$

because if this supremum were less than $\kappa\left(a^{*}\right)$, then Lemma 5 would imply that $\lambda \operatorname{ex~} \mathrm{s}(e, x) \in N\left(\mathfrak{a}^{*}\right)$ is recursive in $\mathfrak{a}^{*}$ which is easily seen to be absurd. Thus

$$
\begin{aligned}
z \in N\left(\mathfrak{a}^{*}\right) & \equiv(E e)(E x)\left[\left.\{e\}\left(\mathfrak{a}^{*}, x\right) \downarrow \&|z|\right|^{*} \leqq|\mathrm{~s}(e, x)|^{\mathfrak{a}^{*}}\right] \\
& \equiv(E e)(E x)\left[\{e\}\left(\mathfrak{a}^{*}, x\right) \downarrow \& \phi\left(\mathfrak{b}^{*}, z, \tau, \mathrm{s}(e, x), \tau\right) \simeq 0\right],
\end{aligned}
$$

which is r.e. in ${ }^{3} E$ by (1) of the theorem.

RemarK. Theorem 9 in $\S 6$ implies that $\lambda z \mathfrak{a} z \in N(\mathfrak{a})$ is also r.e. in ${ }^{3} \mathbf{E}$.

The most interesting consequences of Theorems 5 and 6 are several choice axioms which hold for predicates r.e. in ${ }^{3} \mathbf{E}$. Again, these results are inspired by Gandy's similar theorems for type 2 in [2].

In the equivalence of Theorem 8 we use the restricted quantifiers $(E \alpha)_{\mathfrak{c}},(E \mathrm{~g})_{\mathfrak{c}}$; these are to be read "there exists an $\alpha$ recursive in c," "there exists a $g$ recursive in c." We use " $g$ " as a variable for functions or functionals with arguments of types $\leqq 3$.

THEOREM 8. Let $\mathrm{c}, \mathrm{d}$ be lists of variables of types $\leqq 3$, for each of the equivalences below let $P$ be a predicate in the indicated variables which is r.e. in ${ }^{3} \mathbf{E}$.

(1) $(\delta)(E y) P(\mathfrak{c}, \delta, y) \equiv(E \mathrm{~g})_{\mathfrak{c}}(\mathrm{d}) P(\mathrm{c}, \mathrm{d}, \mathrm{g}(\mathrm{d}))$.

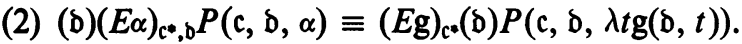

(Particular cases of (1) and (2) are:

(3) $(x)(E y) P(c, x, y) \equiv(E \alpha)_{c^{*}}(x) P(c, x, \alpha(x))$,

(4) $(x)(E \alpha)_{c} P(c, x, \alpha) \equiv(E \alpha)_{c^{*}}(x) P(c, x, \lambda t \alpha(\langle x, t\rangle))$.) 
Proof. The implications from right to left are trivial. To prove (1) and (2) from left to right, let $\mathfrak{c}=\mathfrak{a}, \boldsymbol{x}$ as usual, and pick $e$ in each case so that

$$
P(\mathfrak{c}, \mathfrak{b},-) \equiv\{e\}\left(\mathfrak{c}^{*}, \mathfrak{b},-\right) \downarrow
$$

(1) Put $\mathrm{g}(\mathfrak{D}) \simeq \nu(\mathfrak{c}, \mathfrak{b}, e)$; now (I) implies that $\mathrm{g}(\mathfrak{D})$ is completely defined, recursive in $c^{*}$, and satisfies the right-hand side of (1).

(2) We compute,

$$
\begin{aligned}
& (\mathfrak{b})(E \alpha)_{\mathfrak{c}^{*}, \mathfrak{D}} P(\mathfrak{c}, \mathfrak{d}, \alpha) \rightarrow(\mathfrak{D})(E e)\left[(t)\left[\{e\}\left(\mathfrak{c}^{*}, \mathfrak{b}, t\right) \downarrow\right] \& P\left(\mathfrak{c}, \mathfrak{b}, \lambda t\{e\}\left(\mathfrak{c}^{*}, \mathfrak{d}, t\right)\right)\right] \\
& \rightarrow(\mathfrak{b})(E e)\left[(t)\left[\{e\}\left(\mathfrak{c}^{*}, \mathfrak{d}, t\right) \downarrow\right] \&(\beta)\left[\beta=\lambda t\{e\}\left(\mathfrak{c}^{*}, \mathfrak{b}, t\right) \rightarrow P(\mathfrak{c}, \mathfrak{b}, \beta)\right]\right] .
\end{aligned}
$$

We can easily show that the predicate in brackets is r.e. in ${ }^{3} \mathbf{E}$, using the Substitution Lemma and the fact that the class of predicates r.e. in ${ }^{3} \mathbf{E}$ is closed under universal (number or function) quantification and conjunction. Thus (1) applies, and there exists a functional $g(\mathbb{D})$, recursive in $\mathrm{c}^{*}$, such that

$$
(\mathrm{b})\left[(t)\left[\{\mathrm{g}(\mathrm{D})\}\left(\mathrm{c}^{*}, \mathrm{D}, t\right) \downarrow\right] \& P\left(\mathfrak{c}, \mathrm{D}, \lambda t\{\mathrm{~g}(\mathrm{D})\}\left(\mathrm{c}^{*}, \mathrm{~d}, t\right)\right)\right] .
$$

We obtain then a functional $g(\delta, t)$ that satisfies the right-hand side of $(2)$ by putting

$$
\mathrm{g}(\mathrm{D}, t) \simeq\{\mathrm{g}(\mathrm{D})\}\left(\mathrm{c}^{*}, \mathrm{~d}, t\right) .
$$

COROllary 8.1. For $i=1,2$, put $z \in N_{i}\left(\mathfrak{a}^{*}\right) \equiv z \in N\left(\mathfrak{a}^{*}\right) \&(w)\left[|w|^{\mathfrak{a}^{*}}<|z|^{\mathfrak{a}^{*}} \rightarrow_{i} \mathbf{G}_{z}\right]$ is not recursive in ${ }_{i} \mathbf{G}_{w} \&(w)\left[|w|^{*}=|z|^{*} \rightarrow z \leqq w\right]$.

(1) The predicate $\lambda z \mathfrak{a} z \in N_{\mathfrak{i}}\left(\mathfrak{a}^{*}\right)$ is r.e. in ${ }^{3} \mathbf{E}$.

(2) The set $N_{i}\left(\mathfrak{a}^{*}\right)$ is linearly ordered by $\lambda z w|z|_{c}^{a^{*}} \leqq|w|_{c}^{a^{*}}$ with order-type $\omega_{1}\left(\mathfrak{a}^{*}\right)$.

Proof. (1) follows easily from Corollary 7.1, Lemma 3 and the remark that the predicate " $\mathbf{F}$ is recursive in $\mathbf{G}$ " is hyperanalytic.

To prove (2) by contradiction, assume that the ordering on $N_{i}\left(a^{*}\right)$ is similar to an initial segment of $\omega_{1}\left(\mathfrak{a}^{*}\right)$, say $\left\{|w|_{c}^{\alpha^{*}}:|w|_{c}^{a^{*}}<|z|_{c}^{a^{*}}\right\}$. Put

$$
\begin{aligned}
P(\mathfrak{a}, x, y) & \equiv\left\{\text { not }\left(|x|_{\mathrm{c}}^{a^{*}}<|z|_{\mathrm{c}}^{\mathfrak{a}^{*}}\right) \& y=0\right\} \\
& \vee\left[|x|_{\mathrm{c}}^{\mathfrak{a}^{*}}<|z|_{\mathrm{c}}^{\mathfrak{a}^{*}} \& y \in N_{i}\left(\mathfrak{a}^{*}\right)\right.
\end{aligned}
$$

$\&(\alpha)\left[\alpha\right.$ is a mapping from $\left\{u:|u|_{c}^{a^{*}} \leqq|x|_{c}^{a^{*}}\right\}$ into $\left\{v: v \in N_{i}\left(a^{*}\right) \&|v|_{c}^{a^{*}} \leqq|y|_{c}^{a^{*}}\right\}$ which preserves the ordinal ordering $\rightarrow \alpha(x)=y]$ ].

It is easy to verify that $\left\{v:\left.v \in N_{i}\left(a^{*}\right) \&|v|\right|_{c} ^{\mathfrak{a}^{*}} \leqq|y|_{\mathrm{c}}^{\mathfrak{a}^{*}}\right\}$ is hyperanalytic in $\mathfrak{a}$, uniformly for $y \in N_{i}\left(\mathfrak{a}^{*}\right)$, and hence that $P(\mathfrak{a}, x, y)$ is r.e. in ${ }^{3} \mathbf{E}$. Since, obviously, $(x)(E y) P(\mathfrak{a}, x, y)$, Theorem 8 implies that for some $\alpha$, hyperanalytic in $\mathfrak{a}$,

$$
(x) P(\mathfrak{a}, x, \alpha(x)) \text {. }
$$

But then

$$
y \in N_{i}\left(\mathfrak{a}^{*}\right) \equiv(E x)\left[|x|_{c}^{a^{*}}<|z|_{c}^{a^{*}} \& \alpha(x)=y\right],
$$

so that $N_{i}\left(\mathfrak{a}^{*}\right)$ is hyperanalytic in $\mathfrak{a}$, which is easily seen to be absurd. 
ReMARK. The sequence of predicates ${ }_{i} \mathbf{G}_{z}\left(z \in N_{i}\left(\mathrm{a}^{*}\right)\right)$ provides a strictly increasing sequence of degrees of length $\omega_{1}\left(\mathfrak{a}^{*}\right)$, which is indexed by a set r.e. in $\mathfrak{a}^{*}$ and exhausts the $i$-section of $\mathfrak{a}^{*}$.

6. Normal forms for predicates r.e. in ${ }^{3} E$. One of the main results of RFI is the Representation Theorem XXVIII, which asserts (for type 3) that there exist primitive recursive predicates $L(e, \mathfrak{c}, w, \alpha, \mathbf{F})$ and $M(e, \mathfrak{c}, w, \alpha, \mathbf{F})\left({ }^{4}\right)$, such that

$$
\begin{aligned}
& \{e\}(\mathfrak{c}) \simeq w \equiv(E \mathbf{F})(\alpha) L(e, \mathfrak{c}, w, \alpha, \mathbf{F}), \\
& \{e\}(\mathfrak{c}) \simeq w \equiv(\mathbf{F})(E \alpha) M(e, \mathfrak{c}, w, \alpha, \mathbf{F}) .
\end{aligned}
$$

In this section we shall outline proofs of strengthened versions of these theorems, in which we specify bases (in the sense of [5]) for the quantifiers $(E \mathbf{F}),(\mathbf{F})$ above (our $L$ and $M$ however will be analytic). As corollaries we show that the class of predicates r.e. in ${ }^{3} \mathbf{E}$ is closed under restricted functional quantification $(E \mathbf{F})_{c}$, but not closed under unrestricted function quantification $(E \alpha)$.

THEOREM 9. (1) If $R(\mathfrak{c}, \mathbf{F})$ is hyperanalytic, then $(E \mathbf{F})_{\mathfrak{c}} R(\mathfrak{c}, \mathbf{F})$ is r.e. in ${ }^{3} \mathbf{E}$.

(2) If $P(\mathfrak{c})$ is r.e. in ${ }^{3} \mathbf{E}$, then there exists an analytic $R(\mathfrak{c}, \mathbf{F})$ such that

$$
P(\mathfrak{c}) \equiv(E \mathbf{F}) R(\mathfrak{c}, \mathbf{F}) \equiv(E \mathbf{F})_{\mathfrak{c}} R(\mathbf{c}, \mathbf{F}) .
$$

$\left(\right.$ Thus $P(c)$ is r.e. in ${ }^{3} \mathbf{E}$, if and only if it is expressible in the form $(E \mathbf{F})_{c^{*}} R(\mathfrak{c}, \mathbf{F})$, with analytic $R$.)

Proof. (1) We compute

$$
(E \mathbf{F})_{\mathfrak{c}^{*}} R(\mathfrak{c}, \mathbf{F}) \equiv(E e)\left[(\alpha)\left[\{e\}\left(\mathfrak{c}^{*}, \alpha\right) \downarrow\right] \& R\left(\mathfrak{c}, \lambda \alpha\{e\}\left(\mathrm{c}^{*}, \alpha\right)\right)\right] ;
$$

now the Substitution Lemma implies that the predicate in brackets is r.e. in ${ }^{3} \mathbf{E}$, hence $(E \mathbf{F})_{c^{*}} R(\mathbf{c}, \mathbf{F})$ is r.e. in ${ }^{3} \mathbf{E}$ by (1) of Theorem 7.

To prove (2), we shall define an analytic predicate $L\left(\tau^{3}, \tau^{2}, \tau, z, \mathbf{F}\right)$, such that in the notation of $\S 2$,

(i) $z \in N(\tau) \equiv(E \mathbf{F}) L\left(\tau^{3}, \tau^{2}, \tau, z, \mathbf{F}\right)$

$$
\equiv(E \mathbf{F})\left[\mathbf{F} \text { is recursive in }{ }^{3} \mathbf{E}, \tau^{3}, \tau^{2}, \tau \& L\left(\tau^{3}, \tau^{2}, \tau, z, \mathbf{F}\right)\right] \text {; }
$$

the result will then follow by (A).

The construction is a lengthy but straightforward analysis of the transfinite induction for $z \in N(\tau)$, so we shall omit many of the details.

(ii) We define a predicate $P(u, \alpha, v, \beta, \delta)$ by the disjunction of the following seven clauses. $(P(u, \alpha, v, \beta, \delta)$ will assert that, if $v \in N(\beta)$, then $u \in N(\alpha)$ and $u$ in $N(\alpha)$ is an immediate predecessor of $v$ in $N(\beta)$, relative to $\delta$.) (Notation: $q=w=(v)_{1}, e=(v)_{2}$.)

(1) $v=\langle 1, q\rangle \& u=\langle 1, q\rangle$.

(2) $v=\langle 2,0\rangle \& u=\langle 2,0\rangle$.

(3) $v=\langle 3, w\rangle \& u=w \& a=\beta$.

(4) $v=\langle 4, w, e\rangle \& \alpha=\beta \&[(\mathrm{a}) \vee(\mathrm{b}) \vee(\mathrm{c})]$, where
(a) $u=w$.
(b) $\left[\lambda t\{e\}^{\delta}(t)\right.$ is not completely defined] \& $u=0$.
(c) $\left[\lambda t\{e\}^{\delta}(t)\right.$ is completely defined] \& $(E t)\left[u=\{e\}^{\delta}(t)\right]$. 
(5) $v=\langle 5, w, e\rangle \&[(\mathrm{~d}) \vee(\mathrm{e})]$, where:

(d) [e does not define a $\beta$-recursive functional $] \& u=0$.

(e) [e defines a $\beta$-recursive functional $\{e\}(\gamma, \beta)] \& u=w \&(E \gamma)[\alpha=\{e\}(\gamma, \beta)]$.

(6) $v=\langle 6, w, e\rangle \&[(\mathrm{f}) \vee(\mathrm{g})]$, where:

(f) $\left[\lambda t\{e\}^{\beta}(t)\right.$ is not completely defined $] \& u=0$.

(g) $\left[\lambda t\{e\}^{\beta}(t)=\gamma\right.$ is completely defined] \& $u=w \& \alpha=\gamma$.

(7) [ $v$ does not satisfy any of the case hypotheses for (1)-(6)] \& $u=0$.

(iii) Let $\mathbf{F}(z, \tau)$ and $\mathbf{G}(z, \tau, t)$ be variables for functionals of the indicated list of variables. We define a predicate $S(\mathbf{F}, \mathbf{G})$ by the conjunction of the following eight clauses. (We suppress in the notation the dependence of $S(F, G)$ on $\tau^{3}, \tau^{2}$.)

(1) $\mathbf{F}(\langle 1, q\rangle, \tau)=0 \& \mathbf{G}(\langle 1, q\rangle, \tau, t)=q$.

(2) $\mathbf{F}(\langle 2,0\rangle, \tau)=0 \& \mathbf{G}(\langle 2,0\rangle, \tau, t)=\tau(t)$.

(3) $\mathbf{F}(\langle 3, w\rangle, \tau)=0 \rightarrow\left[\mathbf{F}(w, \tau)=0 \& \mathbf{G}(\langle 3, w\rangle, \tau, t)=\tau^{2}(\lambda u \mathbf{G}(w, \tau, u))\right]$.

(4) $\mathbf{F}(\langle 4, w, e\rangle, \tau)=0 \rightarrow\left[\mathbf{F}(w, \tau)=0 \&\left[\delta=\lambda u \mathbf{G}(w, \tau, u) \rightarrow\left[\lambda t\{e\}^{\delta}(t)\right.\right.\right.$ is completely defined] \& $\left.\left.(t) \mathbf{F}\left(\{e\}^{\delta}(t), \tau\right)=0 \& \mathbf{G}(\langle 4, w, e\rangle, \tau, t)=\mathbf{G}\left(\{e\}^{\delta}(t), \tau, t\right)\right]\right]$.

(5) $\mathbf{F}(\langle 5, w, e\rangle, \tau)=0 \rightarrow[e$ defines a $\tau$-recursive functional $\{e\}(\gamma, \tau)]$ $\left.\&(\gamma)[\mathbf{F}(w,\{e\}(\gamma, \tau))=0] \& \mathbf{G}(\langle 5, w, e\rangle, \tau, t)=\tau^{3}(\lambda \gamma \mathbf{G}(w,\{e\}(\gamma, \tau), t))\right]$.

(6) $\mathbf{F}(\langle 6, w, e\rangle, \tau)=0 \rightarrow\left[\gamma=\lambda t\{e\}^{\tau}(t)\right.$ is completely defined \& $\mathbf{F}(w, \gamma)=0$ $\& \mathbf{G}(\langle 6, w, e\rangle, \tau, t)=\mathbf{G}(w, \gamma, t)]$.

(7) If $x$ is not in any of the forms $\langle 2,0\rangle,\langle 1, q\rangle,\langle 3, w\rangle,\langle 4, w, e\rangle,\langle 5, w, e\rangle$, $\langle 6, w, e\rangle$, then $\mathbf{F}(x, \tau) \neq 0$.

(8) (Well-ordering clause.) For an arbitrary function $\beta$, put

$$
\begin{gathered}
\beta_{n}=\lambda t \beta(\langle n, t\rangle) . \\
(\alpha)(\beta)\left[(x)\left[\mathbf{F}\left(\alpha(x), \beta_{x}\right)=0 \& P\left(\alpha(x+1), \beta_{x+1}, \alpha(x), \beta_{x}, \lambda t \mathbf{G}\left((\alpha(x))_{1}, \beta_{x+1}, t\right)\right)\right]\right. \\
\left.\rightarrow(E x)\left[\alpha(x)=\langle 2,0\rangle \vee \alpha(x)=\left\langle 1,(\alpha(x))_{1}\right\rangle\right]\right] .
\end{gathered}
$$

We now claim:

(iv) $z \in N(\tau) \rightarrow(E \mathbf{F})(E \mathbf{G})\left[\mathbf{F}, \mathbf{G}\right.$ are recursive in ${ }^{3} \mathbf{E}, \tau^{3}, \tau^{2}, \tau, \& S(\mathbf{F}, \mathbf{G})$ $\& \mathbf{F}(z, \tau)=0]$.

(v) $(E \mathbf{F})(E \mathbf{G})[S(\mathbf{F}, \mathbf{G}) \& \mathbf{F}(z, \tau)=0] \rightarrow z \in N(\tau)$.

From these two equivalences we can obtain (i) and then complete the proof of the theorem by routine contractions of variables.

Proof of (iv). If $z \in N(\tau)$, put

$$
\begin{aligned}
\mathbf{F}(x, \gamma) & =0 & & \text { if }|x|^{\gamma} \leqq|z|^{\imath}, \\
& =1 & & \text { otherwise, } \\
\mathbf{G}(x, \gamma, t) & =\mathrm{f}_{x}^{\gamma}(t) & & \text { if }|x|^{\gamma} \leqq|z|^{\tau} \\
& =0 & & \text { otherwise. }
\end{aligned}
$$

Lemma 3 and Theorem 2 imply that $\mathbf{F}$ and $\mathbf{G}$ are recursive in ${ }^{3} \mathbf{E}, \tau^{3}, \tau^{2}, \tau$, and of course $\mathbf{F}(z, \tau)=0$. 
The verification of the first seven clauses of $S(\mathbf{F}, \mathbf{G})$ is immediate. To verify clause (8), we prove by cases on the definition of $P(u, \alpha, v, \beta, \delta)$, that for each $u, v, \alpha, \beta$,

$\left[P\left(u, \alpha, v, \beta, \lambda t \mathbf{G}\left((v)_{1}, \alpha, t\right)\right) \& \mathbf{F}(v, \beta)=0\right]$

$$
\rightarrow\left[v=\langle 2,0\rangle \vee v=\left\langle 1,(v)_{1}\right\rangle\right] \vee\left[|u|^{\alpha}<|v|^{\beta}\right] .
$$

(For example, if $v=\langle 4, w, e\rangle$, then $\alpha=\beta$ and $u$ must satisfy one of (a), (b), (c) with $\delta=\lambda t \mathbf{G}(w, \alpha, t)=\lambda t \mathrm{f}_{w}^{\alpha}(t)$. Since $\mathbf{F}(v, \beta)=0, v \in N(\beta)$, hence $w \in N(\alpha)$ and (b) cannot be true; it is then evident that whether (a) or (c) holds, $|u|^{\alpha}<|v|^{\beta}$.) Clause (8) then follows, since we cannot have an infinite decreasing sequence of ordinals

$$
|\alpha(0)|^{\beta_{0}}>|\alpha(1)|^{\beta_{1}}>\cdots \text {. }
$$

Proof of (v). Assume that $S(\mathbf{F}, \mathbf{G}) \& \mathbf{F}(z, \tau)=0$. Consider the set $\mathscr{T}$ of all finite sequences $\left\{\left(u_{0}, \beta_{0}\right), \ldots,\left(u_{x}, \beta_{x}\right)\right\}$ of pairs of numbers and functions such that

$$
\begin{array}{r}
u_{0}=z \& \beta_{0}=\tau \&(s)_{s<x} P\left(u_{s+1}, \beta_{s+1}, u_{s}, \beta_{s}, \lambda t \mathbf{G}\left(\left(u_{s}\right)_{1}, \beta_{s+1}, t\right)\right) \\
\&(s)_{s \leqq x} \mathbf{F}\left(u_{s}, \beta_{s}\right)=0 .
\end{array}
$$

We can think of $\mathscr{T}$, partially ordered under extension of finite sequences, as a tree, where at each node $\left\{\left(u_{0}, \beta_{0}\right), \ldots,\left(u_{x}, \beta_{x}\right)\right\}$ there may be one, countably many or uncountably many choices for $\left(u_{x+1}, \beta_{x+1}\right)$, depending on the prime number expansion of $u_{x}$.

Consider the predicate of sequences of $\mathscr{T}$,

$$
u_{x} \in N\left(\beta_{x}\right) \& \mathbf{G}\left(u_{x}, \beta_{x}, \mathrm{t}\right)=\mathrm{f}\left(\beta_{x}, u_{x} ; t\right) .
$$

Clause (8) of the definition of $S(\mathbf{F}, \mathbf{G})$ asserts that this predicate has the wellfounded property on $\mathscr{T}$, i.e., if we follow any branch along the tree, we must meet a node where (vi) holds. We shall prove that (vi) holds for all sequences in $\mathscr{T}$ (and hence $z \in N(\tau)$ ) by bar induction on $\mathscr{T}$ (as in [10]). (One may easily rephrase the proof as a classical proof by contradiction.)

Basis of bar induction:

$$
\begin{aligned}
(\alpha)(\beta)\left[( x ) \left\{\left(\alpha(0), \beta_{0}\right),\right.\right. & \left.\ldots,\left(\alpha(x), \beta_{x}\right)\right\} \in \mathscr{T} \\
& \left.\rightarrow(E k)(x)_{x \geqq k}\left[\alpha(x) \in N\left(\beta_{x}\right) \& \mathbf{G}\left(\alpha(x), \beta_{x}, t\right)=\mathrm{f}\left(\beta_{x}, \alpha(x) ; t\right)\right]\right] .
\end{aligned}
$$

This is immediate from clause (8) of definition (ii) and clauses (1) and (2) of definitions (ii) and (iii).

Ind. step of bar induction: Let $\left\{\left(u_{0}, \beta_{0}\right), \ldots,\left(u_{x}, \beta_{x}\right)\right\}$ be a sequence in $\mathscr{T}$. We must show $u_{x} \in N\left(\beta_{x}\right) \& \mathbf{G}\left(u_{x}, \beta_{x}, \mathrm{t}\right)=\mathrm{f}\left(\beta_{x}, u_{x} ; t\right)$, utilizing the following:

Ind. hypothesis: if $\left(u_{x+1}, \beta_{x+1}\right)$ is any pair such that

$$
\left\{\left(u_{0}, \beta_{0}\right), \ldots,\left(u_{x}, \beta_{x}\right),\left(u_{x+1}, \beta_{x+1}\right)\right\} \in \mathscr{T} \text {, }
$$

then $u_{x+1} \in N\left(\beta_{x+1}\right) \& \mathbf{G}\left(u_{x+1}, \beta_{x+1}, t\right)=\mathrm{f}\left(\beta_{x+1}, u_{x+1} ; t\right)$. Since $\mathbf{F}\left(u_{x}, \beta_{x}\right)=0$, we know that $u_{x}$ must be in one of the forms $\langle 2,0\rangle,\langle 1, q\rangle$, etc., and hence in the 
definition of $\mathbf{F}\left(u_{x}, \beta_{x}\right)$ one of the first six clauses must apply. We only treat one of the cases, the others being similar.

Clause (4). $u_{x}=\langle 4, w, e\rangle$. First choose $u_{x+1}=w, \beta_{x+1}=\beta_{x}$; clearly

$$
\mathbf{F}\left(w, \beta_{x}\right)=0 \& P\left(w, \beta_{x}, u_{x}, \beta_{x}, \lambda t \mathbf{G}\left(w, \beta_{x}, t\right)\right),
$$

so the sequence $\left\{\left(u_{0}, \beta_{0}\right), \ldots,\left(u_{x}, \beta_{x}\right),\left(w, \beta_{x}\right)\right\} \in \mathscr{T}$ and by ind. hyp.

$$
w \in N\left(\beta_{x}\right) \& \mathbf{G}\left(w, \beta_{x}, \mathrm{t}\right)=\mathbf{f}\left(\beta_{x}, w ; t\right) .
$$

Moreover, if $\delta=\lambda t \mathbf{G}\left(w, \beta_{x}, t\right)$, then $\lambda t\{e\}^{\delta}(t)$ is comp. defined and for each $t$, $\mathbf{F}\left(\{e\}^{\delta}(t), \beta_{x}\right)=0$. Thus for each $t$, if we choose $u_{x+1}=\{e\}^{\delta}(t), \beta_{x+1}=\beta_{x}$, we have $\left\{\left(u_{0}, \beta_{0}\right), \ldots,\left(u_{x}, \beta_{x}\right),\left(\{e\}^{\delta}(t), \beta_{x}\right)\right\} \in \mathscr{T}$, so by ind. hyp.

$$
\{e\}^{\delta}(t) \in N\left(\beta_{x}\right) \& \mathbf{G}\left(\{e\}^{\delta}(t), \beta_{x}, t\right)=\mathrm{f}\left(\beta_{x},\{e\}^{\delta}(t) ; t\right) .
$$

This implies $\langle 4, w, e\rangle \in N\left(\beta_{x}\right)$, and clause (4) of definition (iii) implies that

$$
\mathbf{G}\left(\langle 4, w, e\rangle, \beta_{x}, t\right)=\mathrm{f}\left(\beta_{x},\langle 4, w, e\rangle ; t\right) .
$$

REMARK. One half of Kleene's Representation Theorem follows from (2) of Theorem 9 if we remark that $\{e\}(c) \simeq w$ is r.e. in ${ }^{3} \mathbf{E}$ and absorb in the usual way all but one of the function quantifiers in the prefix of the analytic predicate $R(\mathfrak{c}, F)$ in the quantifier $(E \mathbf{F})$.

Corollary 9.1. (1) If $P(\mathfrak{c}, \alpha)$ and $Q(\mathfrak{c}, \mathbf{F})$ are r.e. in ${ }^{3} \mathbf{E}$, then so are $(E \alpha)_{\mathfrak{c}} P(\mathfrak{c}, \alpha)$ and $(E \mathbf{F})_{\mathfrak{c}} P(\mathfrak{c}, \mathbf{F})$.

(2) In the notation of Theorem 8 , if $P(c, \delta, \mathbf{F})$ is r.e. in ${ }^{3} \mathbf{E}$, then

$$
(\mathfrak{b})(E \mathbf{F})_{\mathfrak{c}^{\natural}, \mathfrak{D}} P(\mathfrak{b}, \mathfrak{c}, \mathbf{F}) \equiv(E g)_{\mathfrak{c}^{*}}(\mathfrak{d}) P(\mathfrak{c}, \delta, \lambda \alpha \mathrm{g}(\mathfrak{c}, \delta, \alpha)) \text {. }
$$

Proof. (1) By (2) of Theorem 9, there exists an analytic predicate $R(\mathfrak{c}, \mathbf{F}, \mathbf{G})$, such that

$$
Q(\mathfrak{c}, \mathbf{F}) \equiv(E \mathbf{G})_{\mathfrak{c}^{*}, \mathbf{F}} R(\mathfrak{c}, \mathbf{F}, \mathbf{G})
$$

hence

$$
(E \mathbf{F})_{\mathfrak{c}^{*}} Q(\mathfrak{c}, \mathbf{F}) \equiv(E \mathbf{F})_{\mathfrak{c}^{*}}(E \mathbf{G})_{\mathfrak{c}^{*}, \mathbf{F}} R(\mathfrak{c}, \mathbf{F}, \mathbf{G}) \equiv(E \mathbf{F})_{\mathfrak{c}^{*}} R\left(\mathfrak{c},(\mathbf{F})_{0},(\mathbf{F})_{1}\right),
$$

which is r.e. in ${ }^{3} \mathbf{E}$ by (1) of Theorem 9.

(2) $(\mathfrak{b})(E \mathbf{F})_{\mathfrak{c}^{*}, \mathfrak{d}} P(\mathfrak{b}, \mathfrak{c}, \mathbf{F}) \rightarrow(\mathfrak{b})(E e)\left[(E \mathbf{F})_{\mathfrak{c}^{*} \mathfrak{b}}\left[(\alpha)\left[\{e\}\left(\mathfrak{c}^{*}, \mathfrak{d}, \alpha\right) \simeq \mathbf{F}(\alpha)\right] \& P(\mathfrak{c}, \delta, \mathbf{F})\right]\right]$.

The predicate in brackets is r.e. in ${ }^{3} \mathbf{E}$, by (1) of Theorem 9, hence by Theorem 8,

$$
(E \mathrm{~g})_{\mathfrak{c}^{*}}(\delta) P\left(\mathfrak{c}, \delta, \lambda \alpha\{\mathrm{g}(\delta)\}\left(\mathfrak{c}^{*}, \delta, \alpha\right)\right) \text {. }
$$

Put then

$$
\mathrm{g}(\mathfrak{c}, \mathfrak{b}, \alpha) \simeq\{\mathrm{g}(\mathfrak{d})\}\left(\mathfrak{c}^{*}, \mathfrak{d}, \alpha\right)
$$

COROllary 9.2. A predicate $P(\mathfrak{c})$ is hyperanalytic if and only if it can be expressed in both forms $(E \mathbf{F})_{c^{*}} R(\mathfrak{c}, \mathbf{F}),(\mathbf{F})_{c^{*}} Q(\mathfrak{c}, \mathbf{F})$, with analytic $R, Q$.

Proof. Immediate from Theorems 9 and 7. 
TheORem 10. If $P(\mathfrak{c})$ is r.e. in ${ }^{3} \mathbf{E}$, then there exists a predicate $Q(\mathfrak{c}, \alpha)$, r.e. in ${ }^{3} \mathbf{E}$, such that

$$
P(\mathfrak{c}) \equiv(\alpha) \bar{Q}(\mathfrak{c}, \alpha) .
$$

Proof. We shall define a predicate $Q\left(\tau^{3}, \tau^{2}, \tau, \alpha, z\right)$, r.e. in ${ }^{3} \mathbf{E}$, and prove

$$
z \in N(\tau) \equiv(\alpha) \bar{Q}\left(\tau^{3}, \tau^{2}, \tau, \alpha, z\right)
$$

from this the theorem follows by (A).

Let $P(u, \alpha, v, \beta, \delta)$ be the analytic predicate defined in the proof of Theorem 9 , let $\mathrm{p}(z)$ be the function of Theorem 2. By the Substitution Lemma, there exists a predicate $R_{1}\left(\tau^{3}, \tau^{2}, \tau, u, \alpha, v, \beta\right)$, partial recursive in ${ }^{3} \mathbf{E}$, such that

for

$$
P(u, \alpha, v, \beta, \delta) \equiv R_{1}\left(\tau^{3}, \tau^{2}, \tau, u, \alpha, v, \beta\right)
$$

$$
\delta=\lambda t\left\{\mathrm{p}\left((v)_{1}\right)\right\}\left(\tau^{3}, \tau^{2}, \tau, t\right),
$$

when $\delta$ is completely defined. Put

$$
Q(u, \alpha, v, \beta) \equiv[\delta \text { is comp. defined }] \& R_{1}\left(\tau^{3}, \tau^{2}, \tau, u, \alpha, v, \beta\right),
$$

where we are suppressing the dependence of $Q$ on $\tau^{3}, \tau^{2}, \tau$. Evidently $Q$ is r.e. in ${ }^{3}$ E. We claim

(ii) $z \in N(\tau) \rightarrow(\alpha)(\beta)\left[\alpha(0)=z \& \beta_{0}=\tau \&(x) Q\left(\alpha(x+1), \beta_{x+1}, \alpha(x), \beta_{x}\right)\right.$

$$
\left.\rightarrow(E x)\left[\alpha(x)=\langle 2,0\rangle \vee \alpha(x)=\left\langle 1,(\alpha(x))_{1}\right\rangle\right]\right],
$$

(iii) $(\alpha)(\beta)\left[\alpha(0)=z \& \beta_{0}=\tau \&(x) Q\left(\alpha(x+1), \beta_{x+1}, \alpha(x), \beta_{x}\right)\right.$

$$
\left.\rightarrow(E x)\left[\alpha(x)=\langle 2,0\rangle \vee \alpha(x)=\left\langle 1,(\alpha(x))_{1}\right\rangle\right]\right] \rightarrow z \in N(\tau) .
$$

Now (i) follows easily from (ii) and (iii), since the predicate in brackets is clearly the negation of a predicate r.e. in ${ }^{3} \mathbf{E}$.

Proof of (ii) (by induction on $z \in N(\tau)$ ). Given $\alpha, \beta$ such that

$$
\alpha(0)=z \& \beta_{0}=\tau \&(x) Q\left(\alpha(x+1), \beta_{x+1}, \alpha(x), \beta_{x}\right),
$$

choose $\alpha^{\prime}, \beta^{\prime}$ such that

$$
\alpha^{\prime}(x)=\alpha(x+1), \quad \beta_{x}^{\prime}=\beta_{x+1} .
$$

Clearly $(x) Q\left(\alpha^{\prime}(x+1), \beta_{x+1}^{\prime}, \alpha^{\prime}(x), \beta_{x}^{\prime}\right)$. Now we verify that since $Q\left(\alpha(1), \beta_{1}, \alpha(0), \beta_{0}\right)$, we must have $\alpha(1) \in N\left(\beta_{1}\right)$, i.e., $\alpha^{\prime}(0) \in N\left(\beta_{0}^{\prime}\right)$; hence by induction hypothesis, $(E x)\left[\alpha^{\prime}(x)=\langle 2,0\rangle \vee \alpha^{\prime}(x)=\left\langle 1,\left(\alpha^{\prime}(x)\right)_{1}\right\rangle\right]$ which easily implies

$$
(E x)\left[\alpha(x)=\langle 2,0\rangle \vee \alpha(x)=\left\langle 1,(\alpha(x))_{1}\right\rangle\right] .
$$

Proof of (iii). Assume the left-hand side of (iii). Consider the tree $\mathscr{T}$ of all finite sequences $\left\{\left(u_{0}, \beta_{0}\right), \ldots,\left(u_{x}, \beta_{x}\right)\right\}$ such that

$$
u_{0}=z \& \beta_{0}=\tau \&(s)_{s<x} Q\left(u_{s+1}, \beta_{s+1}, u_{s}, \beta_{s}\right) .
$$


We prove by bar induction on $\mathscr{T}$, that for every sequence $\left\{\left(u_{0}, \beta_{0}\right), \ldots,\left(u_{x}, \beta_{x}\right)\right\} \in \mathscr{T}$,

$$
u_{x} \in N\left(\beta_{x}\right)
$$

in particular then $z \in N(\tau)$.

Basis of bar induction: This is immediate from the left-hand side of (iii).

Ind. step of bar induction: We must show that $u_{x} \in N\left(\beta_{x}\right)$, under the ind. hyp. that $u_{x+1} \in N\left(\beta_{x+1}\right)$, whenever $\left\{\left(u_{0}, \beta_{0}\right), \ldots,\left(u_{x}, \beta_{x}\right), \quad\left(u_{x+1}, \beta_{x+1}\right)\right\} \in \mathscr{T}$, i.e., whenever $Q\left(u_{x+1}, \beta_{x+1}, u_{x}, \beta_{x}\right)$. Here we take cases on the definition of

$$
Q\left(u_{x+1}, \beta_{x+1}, u_{x}, \beta_{x}\right),
$$

and again we omit all cases save one.

Clause (4). $u_{x}=\langle 4, w, e\rangle$. First take $u_{x+1}=w, \beta_{x+1}=\beta_{x}$; as in the proof of Theorem 9, it follows that $w \in N\left(\beta_{x}\right)$, and hence $\delta=\lambda t\{\mathrm{p}(w)\}\left(\tau^{3}, \tau^{2}, \beta_{x}, t\right)$ is completely defined. If $\lambda t\{e\}^{\delta}(t)$ is not completely defined, put

$$
\begin{aligned}
\alpha^{\prime}(s)=u_{s} & \text { if } s \leqq x, & \beta_{s}^{\prime}=\beta_{s} & \text { if } s \leqq x, \\
=0 & \text { if } s>x, & \beta_{x} & \text { if } s>x,
\end{aligned}
$$

and check that $\alpha^{\prime}, \beta^{\prime}$ satisfy the antecedent of the left-hand side of (iii), but not the consequent. Hence $\lambda t\{e\}^{\delta}(t)$ is completely defined, and for each $t$ we can take $u_{x+1}=\{e\}^{\delta}(t), \beta_{x+1}=\beta_{x}$ and verify that $\left\{\left(u_{0}, \beta_{0}\right), \ldots,\left(u_{x}, \beta_{x}\right),\left(u_{x+1}, \beta_{x+1}\right)\right\} \in \mathscr{T}$. Then by ind. hyp. $\{e\}^{\delta}(t) \in N\left(\beta_{x}\right)$, so $\langle 4, w, e\rangle \in N\left(\beta_{x}\right)$.

COROllary 10.1. If $P(c)$ is r.e. in ${ }^{3} \mathbf{E}$, then there exists an analytic predicate $R(\mathrm{c}, \alpha, \mathbf{F})$, such that

$$
P(\mathfrak{c}) \equiv(\alpha)(\mathbf{F}) R(\mathfrak{c}, \alpha, \mathbf{F}) \equiv(\alpha)(\mathbf{F})_{\mathfrak{c}^{*}, \alpha} R(\mathfrak{c}, \alpha, \mathbf{F}) .
$$

Proof. Immediate from Theorems 9 and 10.

REMARK. The second half of Kleene's Representation Theorem follows from this corollary.

COROLlARY 10.2. There exists a predicate $P(z, \alpha)$, r.e. in ${ }^{3} \mathbf{E}$, such that $(E \alpha) P(z, \alpha)$ is not r.e. in ${ }^{3} \mathbf{E}$.

Proof. The predicate $z \in N\left(\mathfrak{a}^{*}, \alpha\right)\left({ }^{4}\right)$ is r.e. in ${ }^{3} \mathbf{E}$, by Corollary 7.1 ; we show that $(E \alpha)\left[z \in N\left(\mathfrak{a}^{*}, \alpha\right)\right]$ cannot be r.e. in ${ }^{3} \mathbf{E}$.

Let $P(\mathfrak{c})$ be any predicate r.e. in ${ }^{3} \mathbf{E}$. By the theorem,

$$
P(\mathfrak{c}) \equiv(\alpha) \bar{Q}(\mathfrak{c}, \alpha)
$$

for a suitable $Q$, r.e. in ${ }^{3} \mathbf{E}$, and by (A)

for a suitable $e$. Thus

$$
Q(\mathfrak{c}, \alpha) \equiv \mathrm{s}(e, \boldsymbol{x}) \in N\left(\mathfrak{a}^{*}, \alpha\right)
$$

$$
\bar{P}(\mathfrak{c}) \equiv(E \alpha)\left[\mathrm{s}(e, x) \in N\left(\mathfrak{a}^{*}, \alpha\right)\right],
$$

and if $(E \alpha)\left[z \in N\left(\mathfrak{a}^{*}, \alpha\right)\right]$ were r.e. in ${ }^{3} \mathbf{E}, \bar{P}(\mathfrak{c})$ would be r.e. in ${ }^{3} \mathbf{E}$. Since $P(\mathfrak{c})$ was an arbitrary predicate r.e. in ${ }^{3} \mathbf{E}$, this is absurd. 
7. An extension of the hyperanalytic hierarchy. In this section we outline briefly the theory of predicates $P(\mathfrak{c})$ of the form $(E \alpha) R(\mathrm{c}, \alpha)$, with $R$ r.e. in ${ }^{3} \mathbf{E}$. We are mostly interested in the case when $\mathrm{c}$ contains variables of types $\leqq 1$, when this class resembles in many ways the class of $\Sigma_{2}^{1}$ number-theoretic predicates. (A hierarchy of $\Delta_{2}^{1}$ number-theoretic predicates very similar to the hierarchy $\mathscr{D}\left(\mathfrak{a}^{*}\right)$ constructed below, is given in $\$ 16.6$ of [8].)

Definition. (1) Let $a$ be a list of objects of types 2 and 3, $c$ a list of variables of types $\leqq 3\left({ }^{4}\right)$. Let $\mathscr{S}(\mathfrak{a})$ be the class of predicates $P(\mathfrak{c})$ such that for some $R(\mathfrak{c}, \alpha)$, r.e. in $\mathfrak{a}$,

$$
P(\mathfrak{c}) \equiv(E \alpha) R(\mathrm{c}, \alpha) .
$$

Let $\mathscr{P}(\mathfrak{a})$ be the class of predicates $P(\mathfrak{c})$ such that $\bar{P}(\mathfrak{c}) \in \mathscr{S}(\mathfrak{a})$, let

$$
\mathscr{D}(\mathfrak{a})=\mathscr{S}(\mathfrak{a}) \cap \mathscr{P}(\mathfrak{a}) .
$$

A set or function is in $\mathscr{S}(\mathfrak{a}), \mathscr{P}(\mathfrak{a})$ or $\mathscr{D}(\mathfrak{a})$ if its representing predicate is in $\mathscr{S}(\mathfrak{a}), \mathscr{P}(\mathfrak{a})$ or $\mathscr{D}(\mathfrak{a})$ respectively.

(2) $z \in S(\mathfrak{a}) \equiv(E \alpha)[z \in N(\mathfrak{a}, \alpha)]$.

(3) For $z \in S(\mathfrak{a})$, put

$$
\|z\|^{\mathfrak{a}}=\|z\|(\mathfrak{a})=\text { infinum }\left\{|z|^{\mathfrak{a}, \alpha}: z \in N(\mathfrak{a}, \alpha)\right\} .
$$

Put

$$
\lambda(\mathfrak{a})=\operatorname{supremum}\left\{\|z\|^{\mathfrak{a}}: z \in S(\mathfrak{a})\right\} .
$$

In the list of results (i)-(x) below we follow the conventions of $\left({ }^{4}\right)$, so that in particular $\mathfrak{a}^{*}={ }^{3} \mathbf{E}, \mathfrak{a}$.

(i) $\mathscr{S}\left(\mathfrak{a}^{*}\right)$ is closed under the operations \&, $\vee,(E x),(x),(E \alpha)$.

(ii) $\mathscr{D}\left(\mathfrak{a}^{*}\right)$ is closed under the operations \&, $\vee, \neg,(E x),(x),(E \alpha),(\alpha)$ and contains all predicates r.e. in $\mathfrak{a}^{*}$.

Proof. By Theorem 10.

(iii) The predicate $z \in S(\mathfrak{a}, \tau)$ is complete for predicates $P(\tau, x)$ in $\mathscr{S}(\mathfrak{a})$. I.e., for each $P(\tau, x)$ in $\mathscr{S}(\mathfrak{a})$, there exists a primitive recursive $\mathrm{g}(\boldsymbol{x})$ so that

$$
P(\tau, x) \equiv \mathrm{g}(\boldsymbol{x}) \in S(\mathfrak{a}, \tau) .
$$

Proof. By (A).

(iv) $\mathscr{S}\left(\mathfrak{a}^{*}\right)$ does not contain the predicate $z \notin S\left(\mathfrak{a}^{*}, \tau\right)$, and hence $\mathscr{S}\left(\mathfrak{a}^{*}\right)$ is not closed under the operations $\neg,(\alpha)$.

Proof. Assume that $\mathscr{S}\left(\mathfrak{a}^{*}\right)$ contains the predicate $z \notin S\left(\mathfrak{a}^{*}, \tau\right)$. Then $\mathscr{S}\left(\mathfrak{a}^{*}\right)$ $=\mathscr{D}\left(\mathfrak{a}^{*}\right)$, so by (iii) $\mathscr{D}\left(\mathfrak{a}^{*}\right)$ has a complete predicate. However the closure properties (ii) easily imply that $\mathscr{D}\left(\mathfrak{a}^{*}\right)$ cannot have a complete predicate.

By (ii) and Corollary 7.1, the predicate $z \notin N\left(\mathfrak{a}^{*}, \alpha\right)$ is in $\mathscr{S}\left(\mathfrak{a}^{*}\right)$; if $\mathscr{S}\left(\mathfrak{a}^{*}\right)$ were closed under $(\alpha)$, then the predicate $(\alpha)\left[z \notin N\left(a^{*}, \alpha\right)\right] \equiv z \notin S\left(a^{*}\right)$ would be in $\mathscr{S}\left(\mathfrak{a}^{*}\right)$, contradicting the first part of (iv). 
(v) Let $\mathfrak{b}$ be a list of objects of types 2 and 3 , let $\alpha=\alpha_{1}, \ldots, \alpha_{k}, \beta=\beta_{1}, \ldots, \beta_{j}$ be two lists of function variables $\left({ }^{4}\right)$. There exist predicates $L(k, j, z, \alpha, w, \beta)$ and $M(k, j, z, \alpha, w, \beta)$ in $\mathscr{S}\left(\mathfrak{b}^{*}\right)$, such that if $z \in S\left(\mathfrak{b}^{*}, \alpha\right)$, then

$$
\begin{gathered}
\|w\|\left(\mathfrak{b}^{*}, \boldsymbol{\beta}\right) \leqq\|z\|\left(\mathfrak{b}^{*}, \boldsymbol{\alpha}\right) \equiv L(k, j, z, \boldsymbol{\alpha}, w, \boldsymbol{\beta}), \\
\neg\left(\|w\|\left(\mathfrak{b}^{*}, \boldsymbol{\beta}\right) \leqq\|z\|\left(\mathfrak{b}^{*}, \boldsymbol{\alpha}\right)\right) \equiv M(k, j, z, \boldsymbol{\alpha}, w, \boldsymbol{\beta}) .
\end{gathered}
$$

(I.e., the initial segment of ordinals $\|w\|\left(\mathfrak{b}^{*}, \beta\right)$ which are $\leqq\|z\|\left(\mathfrak{b}^{*}, \alpha\right)$ is in $\mathscr{D}\left(\mathfrak{b}^{*}, \alpha\right)$, uniformly for $z \in S\left(\mathfrak{b}^{*}, \alpha\right)$.)

Proof. If $z \in S\left(\mathfrak{b}^{*}, \alpha\right)$, then the following equivalences hold:

$$
\begin{aligned}
\|w\|\left(\mathfrak{b}^{*}, \boldsymbol{\beta}\right) & \leqq\|z\|\left(\mathfrak{b}^{*}, \boldsymbol{\alpha}\right) \\
& \equiv(E \gamma)\left[w \in N\left(\mathfrak{b}^{*}, \boldsymbol{\beta}, \gamma\right) \&(\delta) \neg\left[|z|\left(\mathfrak{b}^{*}, \boldsymbol{\alpha}, \delta\right)<|w|\left(\mathfrak{b}^{*}, \boldsymbol{\alpha}, \gamma\right)\right]\right] . \\
\neg\left[\|w\|\left(\mathfrak{b}^{*}, \boldsymbol{\beta}\right)\right. & \left.\leqq\|z\|\left(\mathfrak{b}^{*}, \boldsymbol{\alpha}\right)\right] \\
& \equiv(E \delta)\left[z \in N\left(\mathfrak{b}^{*}, \boldsymbol{\beta}, \delta\right) \&(\gamma) \neg\left[|w|\left(\mathfrak{b}^{*}, \boldsymbol{\beta}, \gamma\right)<|z|\left(\mathfrak{b}^{*}, \boldsymbol{\alpha}, \delta\right)\right]\right] .
\end{aligned}
$$

Lemma 3 and Corollary 7.4 imply that in each case the predicate in brackets on the right is r.e. in $\mathfrak{b}^{*}$, so the right-hand side is in $S\left(\mathfrak{b}^{*}\right)$.

(vi) (Boundedness.) Let $P(\alpha, x)$ be in $\mathscr{S}\left(\mathfrak{a}^{*}\right)$, let $\mathrm{g}(\boldsymbol{x})$ be a function in $\mathscr{D}\left(\mathfrak{a}^{*}\right)$ such that

$$
P(\alpha, x) \equiv \mathrm{g}(x) \in S\left(\mathfrak{a}^{*}, \alpha\right) .
$$

Then $P(\alpha, x)$ is in $\mathscr{D}\left(\mathfrak{a}^{*}\right)$ if and only if

$$
\text { supremum }\left\{\|\mathrm{g}(\boldsymbol{x})\|\left(\mathfrak{a}^{*}, \boldsymbol{\alpha}\right): P(\boldsymbol{\alpha}, \boldsymbol{x})\right\}<\lambda\left(\mathfrak{a}^{*}\right) .
$$

The proof is similar to that of Lemma 5, using (v) instead of Lemma 3.

(vii) For each $z \in S\left(\mathfrak{a}^{*}\right)$, put

Then

$$
\mathbf{S}_{z}(u, \alpha, v, \beta) \equiv\|v\|\left(\mathfrak{a}^{*}, \beta\right) \leqq\|z\|\left(\mathfrak{a}^{*}\right) \&\|u\|\left(\mathfrak{a}^{*}, \alpha\right) \leqq\|v\|\left(\mathfrak{a}^{*}, \beta\right) .
$$

(1) Each $\mathbf{S}_{z}$ is in $\mathscr{D}\left(\mathfrak{a}^{*}\right)$, uniformly for $z \in S\left(\mathfrak{a}^{*}\right)$.

(2) If $\|z\|^{\mathfrak{a}^{*}}=\|w\| \|^{*}$, then $\mathbf{S}_{z} \equiv \mathbf{S}_{w}$.

(3) If $\|z\|^{\mathfrak{a}^{*}}<\|w\|^{\mathfrak{a}^{*}}$, then $\mathbf{S}_{z}$ is recursive in $\mathbf{S}_{w}$.

(4) If $P(\alpha, x)$ is in $\mathscr{D}\left(a^{*}\right)$, then $P(\alpha, x)$ is recursive in some $\mathbf{S}_{z}$.

The proof is similar to that of Theorem 4, using (vi) instead of Lemma 5.

(viii) (Choice.) Let $P(x, y)$ be in $\mathscr{S}\left(a^{*}\right)$, assume that $(x)(E y) P(x, y)$; then there exists a function $\mathrm{g}(x)$ in $\mathscr{D}\left(\mathfrak{a}^{*}\right)$ such that $(x) P(x, \mathrm{~g}(x))$.

Proof. Using (iii), choose a primitive recursive $\mathrm{f}(x, y)$ such that

and put

$$
P(x, y) \equiv \mathrm{f}(x, y) \in S\left(\mathfrak{a}^{*}\right),
$$

$$
\begin{aligned}
& \mathrm{g}(x)=y \equiv \mathrm{f}(x, y) \in S\left(\mathfrak{a}^{*}\right) \\
& \quad \&(u)\left[\|\mathrm{f}(x, u)\|^{*} \leqq\|\mathrm{f}(x, y)\| \|^{*} \rightarrow\left[\|\mathrm{f}(x, u)\|^{a^{*}}=\|\mathrm{f}(x, y)\| \|^{*} \& y \leqq u\right]\right] .
\end{aligned}
$$

Now (v) easily implies that $\mathrm{g}(x)=y$ is in $\mathscr{S}\left(\mathrm{a}^{*}\right)$; since $\mathrm{g}(x) \neq y \equiv(E z)[z \neq y \& \mathrm{~g}(x)=z]$, the predicate $\mathrm{g}(x)=y$ (and hence the function $\mathrm{g}(x))$ is in $\mathscr{D}\left(\mathfrak{a}^{*}\right)$. 
(ix) For each $\mathfrak{a},\left\{\|z\|^{\mathfrak{a}}: z \in S(\mathfrak{a})\right\}$ is a countable set of ordinals; let $\psi$ be the unique order-preserving function which maps $\left\{\|z\|^{\mathfrak{a}}: z \in S(\mathfrak{a})\right\}$ onto an initial segment of the countable ordinals. Put

(1) $\|z\|_{\mathfrak{c}}^{\mathfrak{a}}=\psi\left(\|z\|^{\mathfrak{a}}\right)(z \in S(\mathfrak{a}))$;

(2) $\omega_{2}^{\mathfrak{a}}=$ supremum $\left\{\|z\|_{\mathfrak{c}}^{\mathfrak{a}}: z \in S(\mathfrak{a})\right\}$;

(3) a countable ordinal $\eta$ is a $\mathscr{D}(\mathfrak{a})$-ordinal if there exists a predicate in $\mathscr{D}(\mathfrak{a})$ which is a well-ordering with order-type $\eta$. Then $\omega_{2}^{a^{*}}$ is the supremum of all $\mathscr{D}\left(\mathfrak{a}^{*}\right)$-ordinals.

Proof. That each $\|z\|_{\mathrm{c}}^{\mathrm{a}^{*}}$ is a $\mathscr{D}\left(\mathfrak{a}^{*}\right)$-ordinal is easily proved from $(\mathbf{v})$ by the method of proof of the first part of Theorem 5.

We prove that $\omega_{2}^{\mathfrak{a}^{*}}$ is not a $\mathscr{D}\left(\mathfrak{a}^{*}\right)$-ordinal by contradiction; assume that $Q(x, y)$ is in $\mathscr{D}\left(\mathfrak{a}^{*}\right)$ and defines a well-ordering with field $D$ and order-type $\omega_{2}^{\mathfrak{a}^{*}}$. Put

$$
\begin{gathered}
P(x, z) \equiv[x \notin D \& z=0] \\
\vee\left[x \in D \& z \in S\left(\mathfrak{a}^{*}\right) \&(E \alpha)[\alpha \text { maps the initial segment of the }\right. \\
\text { ordering defined by } Q \text { up to and including } x \text { into } \\
\left.\left.\left\{w:\|w\|^{*} \leqq\|z\|^{*}\right\} \text { in an order-preserving fashion }\right]\right] .
\end{gathered}
$$

It is easy to verify that $P(x, z)$ is in $\mathscr{S}\left(a^{*}\right)$, and clearly $(x)(E z) P(x, z)$; thus by (viii) there is a $\mathrm{g}(x)$ in $\mathscr{D}\left(\mathfrak{a}^{*}\right)$, so that $(x) P(x, \mathrm{~g}(x))$. Hence

$$
z \in S\left(\mathfrak{a}^{*}\right) \equiv(E x)\left[x \in D \&\|z\|^{*} \leqq\|\mathrm{~g}(x)\| \mathfrak{a}^{*}\right]
$$

which implies that $z \in S\left(\mathfrak{a}^{*}\right)$ is in $\mathscr{D}\left(\mathfrak{a}^{*}\right)$, contradicting (iv).

(x) Put

$$
\begin{aligned}
& z \in S_{2}\left(\mathfrak{a}^{*}\right) \equiv z \in S\left(\mathfrak{a}^{*}\right) \&(w)\left[\|w\|^{*}<\|z\|^{*} \rightarrow \mathbf{S}_{z}\right. \text { is not } \\
& \text { hyperanalytic in } \left.\mathbf{S}_{w}\right] \&(w)\left[\|w\| \mathfrak{a}^{*}=\|z\|^{*} \rightarrow z \leqq w\right] .
\end{aligned}
$$

Then the predicate $z \in S_{2}\left(\mathfrak{a}^{*}\right)$ is in $\mathscr{S}\left(\mathfrak{a}^{*}\right)$ and the set of ordinals $\left\{\|z\|_{\mathrm{c}}^{*}: z \in S_{2}\left(\mathfrak{a}^{*}\right)\right\}$ has order-type $\omega_{2}^{a^{*}}$.

Proof. To prove the first assertion we simply verify that the predicate " $F$ is hyperanalytic in $\mathbf{G}$ " is r.e. in ${ }^{3} \mathbf{E}$, and hence in $\mathscr{D}\left(a^{*}\right)$. The second assertion is proved by the method used in (ix).

8. Comments on results for types other than 3. Suppose that we alter our basic Definition 1 by deleting clauses (5) and (6). It can be verified that the set of functions $\mathrm{f}_{z}^{\tau}(t)(z \in N(\tau))$ coincides then with the set of functions recursive in $\tau^{2}, \tau$. In fact all the subsequent theorems of this paper hold with this modification, and the proofs are somewhat simpler, since there are two fewer cases to worry about. However we obtain nothing in this way (other than the basic characterization) which is not either explicit or implicit in Gandy's [2] and Shoenfield's [9]. (We do think though that our methods are simpler.)

On the other hand one may attempt to extend Definition 1 by adding clauses like (5) and (6) which introduce objects of types $>3$. We believe that in this way one can obtain natural hierarchies for the hyper-(order-r) predicates with variables of types $<r$ (RFII 11.26) for each $r$, but we have not carried out the relevant computations. 


\title{
BIBLIOGRAPHY
}

1. D. A. Clarke, Hierarchies of predicates of finite types, Mem. Amer. Math. Soc. No. 51 (1964), $96 \mathrm{pp}$.

2. R. O. Gandy, General recursive functionals of finite type and hierarchies of functions, mimeographed copy of paper presented at the Sympos. Math. Logic, Univ. of Clermont Ferrand, June 1962. (Similar material was presented by Gandy at the Summer School in Math. Logic, Univ. of Leicester, August 1965, and a paper will appear in the Proceedings of that Summer School.)

3. S. C. Kleene, Introduction to metamathematics, Van Nostrand, New York, 1952.

4. - On the forms of predicates in the theory of constructive ordinals. II, Amer. J. Math. 77 (1955), 405-428.

5. - Quantification of number-theoretic functions, Compositio Math. 14 (1959), 23-40.

6. - Recursive functionals and quantifiers of finite types. I, Trans. Amer. Math. Soc. 91 (1959), 1-52.

7. - Recursive functionals and quantifiers of finite types. II, Trans. Amer. Math. Soc. 108 (1963), 106-142.

8. H. Rogers, Jr., Recursive functions and effective computability, McGraw-Hill, New York.

9. J. R. Shoenfield, A hierarchy for objects of type 2, Abstract 65T-173, Notices Amer. Math. Soc. 81 (1965), 369.

10. C. Spector, Provably recursive functionals of analysis: a consistency proof of analysis by an extension of principles formulated in current intuitionistic mathematics, pp. 1-27, Recursive function theory, Proc. Sympos. Pure Math., Vol. 5, Amer. Math. Soc., Providence, R. I., 1962.

\author{
UNIVERSITy OF CALIFORNIA, \\ Los ANgeles, California
}

